\title{
Review
}

\section{What do eye movements tell us about patients with neurological disorders? - An introduction to saccade recording in the clinical setting -}

\author{
By Yasuo TeraO, ${ }^{* 1, \dagger}$ Hideki FukUdA ${ }^{* 2}$ and Okihide HikOSAKA ${ }^{* 3}$
}

(Communicated by Masanori OTsukA, M.J.A.)

\begin{abstract}
Non-invasive and readily implemented in the clinical setting, eye movement studies have been conducted extensively not only in healthy human subjects but also in patients with neurological disorders. The purpose of saccade studies is to "read out" the pathophysiology underlying neurological disorders from the saccade records, referring to known primate physiology. In the current review, we provide an overview of studies in which we attempted to elucidate the patterns of saccade abnormalities in over 250 patients with neurological disorders, including cerebellar ataxia and brainstem pathology due to neurodegenerative disorders, and what they tell about the pathophysiology of patients with neurological disorders. We also discuss how interventions, such as deep brain stimulation, affect saccade performance and provide further insights into the workings of the oculomotor system in humans. Finally, we argue that it is important to understand the functional significance and behavioral correlate of saccade abnormalities in daily life, which could require eye tracking methodologies to be performed in settings similar to daily life.
\end{abstract}

Keywords: ataxia, basal ganglia, cerebellum, cerebral cortex, parkinsonism, saccade

\section{What are gaze movements, and what can we learn from them?}

The eyes are the windows to the world. We explore the outer world by performing eye movements (usually saccades), and then fixating on objects alternately. ${ }^{1)}$ Intuitively, we implicitly assume that we can acquire the gist of a scene at a single glance, i.e., even in a short period before we have made any eye movements, but this is just an illusion. This becomes clear when we read a text. Unless we gaze at each word (or two words, if one of

*1 Department of Cell Physiology, Kyorin University, Tokyo, Japan.

*2 Neurological Clinic for Children, Tokyo, Japan.

*3 Section of Neuronal Networks, Laboratory of Sensorimotor Research, National Eye Institute, U.S.A.

$\dagger$ Correspondence should be addressed: Y. Terao, Department of Cell Physiology, School of Medicine, Kyorin University, 6-20-2 Shinkawa, Mitaka, Tokyo 181-8611, Japan (e-mail: yteraotky@umin.ac.jp).

Abbreviations: AD: Alzheimer's disease; ADHD: attention deficit hyperactivity disorder; AS: antisaccade; BG: basal ganglia; CFN: cerebellar fastigial nuclei; DBS: deep brain stimulation; DLPFC: dorsolateral prefrontal cortex; EBN: excitatory burst neuron; EOG: electrooculogram; FASD: fetal alcohol spectrum the words is short enough) one at a time, we are not able to get even a rough idea of the meaning of what is written; a "single glance" is not sufficient to grasp everything. This is because only 1-2 degrees of the visual field (central vision) of the retina is capable of high resolution, and we need to use eye movements to attentively examine each word in the text. ${ }^{2)}$ If an eye movement is not possible, we move our gaze by moving the head instead. ${ }^{3)}$ The illusion of getting the "gist" of a scene at a glance may come from our brain's tendency for sparse coding; ${ }^{4)}$ we collect visual information from the "important" parts of the scene

disorder; FEF: frontal eye field; GS: gap saccade; IBN: inhibitory burst neuron; LIP: lateral intraparietal cortex; MGS: memoryguided saccade; MN: motoneuron; MSA-C: multiple system atrophy (cerebellar type); MSA-P: multiple system atrophy (Parkinsonian type); NRTP: nucleus reticularis tegmenti pontis; PD: Parkinson's disease; PEF: parietal eye field; PPC: posterior parietal cortex; PPRF: paramedian pontine reticular formation; PSP: progressive supranuclear palsy; riMLF: rostral interstitial nucleus of the medial longitudinal fasciculus; SC: superior colliculus; SCD: spinocerebellar degeneration; SEF: supplementary eye field; SNc: substantia nigra pars compacta; SNr: substantia nigra pars reticulate; STN: subthalamic nucleus; STN DBS: deep brain stimulation of the subthalamic nucleus; SWJ: square-wave jerk; VGS: visually guided saccade. 
with central vision, but supplement the rest of the information by guessing.

Because we are "visual" animals, the object that the gaze is currently focused on often reflects to some extent what our brain is processing at the moment. This is what we do in our daily lives, i.e., attempting to read another person's mind by looking at his/her gaze.

We are virtually unaware of our own eye movements, although we perform a large number of saccades over the course of 1 day. By contrast, the eyes and their movements are remarkably visible from the outside, i.e., they are the most "visible" part of the brain's processes. As the proverb goes, "the eyes are as eloquent as the tongue". Therefore, by studying how a person's gaze moves, we can at least partly infer what he/she is "thinking" or what mental state he/she is in. ${ }^{5)}$ In a more experimental setting, it is also possible to gain insights into brain function and dysfunction by examining eye movements using various oculomotor paradigms. ${ }^{6), 7)}$

Eye movements consist of two main classes: slow and fast eye movements. ${ }^{6}$ ) Slow movements largely correspond to pursuit, which refers to smooth eye movements that occur when the eyes track a moving stimulus to keep it centered on the fovea. For pursuit to occur, the visual target needs to move at a relatively low speed (30-45 deg/sec in terms of visual angle). By contrast, fast movements correspond mainly to saccades, which are rapid, ballistic eye movements that abruptly change the line of gaze. They do not necessarily require the presence of a visual target and can be made in darkness or according to a memorized target location. Most eye movements performed in daily life when we explore the outer world are also saccades (alternated with fixations), which will be the main subject of this review. Saccades are performed at a rate of $2-6$ times a second, and thus the total number of saccades made within a day amounts to as many as several tens or hundreds of thousands. When properly analyzed, saccades provide a large potential source of information about a subject's neurological condition.

The neural systems involved in eye movement generation have been extensively studied in animals, and the basic organization of the human oculomotor system is known to be very similar. ${ }^{8}$ ) Therefore, by recording eye movements in humans, especially in patients with neurological disorders, and by referring to the normal neurophysiology known from animal studies, we can expect to comprehend what is going on in the brain.

\section{How are saccades studied in humans?}

How do neurologists clinically examine the eye movements of patients? To examine smooth pursuit, they ask a patient to follow their finger as it moves to the left or right and back again, or from top to bottom and back again. Pursuit is a slow, smooth eye movement to capture the target with the fovea (i.e., central vision), and it can be assessed relatively reliably at the bedside. When eye movements are disordered, saccades intervene during attempted pursuit (saccadic pursuit), as is often observed in neurological patients. This is a rather "non-specific" finding with regard to diagnosis.

To assess saccades, neurologists place the index fingers of both hands side by side or on top of the other, alternately bending one by one, and ask the subjects to look at the bent (or extended) finger. This test is used to see whether the saccades are initiated quickly in response to the movements of the fingers. In contrast to pursuit, saccadic eye movements are so fast, reaching an angular velocity of up to 500$600 \mathrm{deg} / \mathrm{s}$ in some cases, that they are usually difficult to evaluate accurately with the naked eye. Thus, we need to record saccades to reliably assess the speed of the saccades, and the latency with which they are initiated.

The prototype method of eye movement recording devised by Yarbus ${ }^{1)}$ involved a suction cup that was attached to the eye. This device is equipped with a small mirror protruding from its side. By projecting light from a candle onto this mirror and burning a light-sensitive paper with its reflection, the trajectory of the gaze can be recorded. Admittedly, this method of recording is painful and irritates the eyes, and it allowed recording only for several minutes at the most, even with the help of a topical ophthalmic anesthetic applied to the eye. Thus, this prototype method was quite impractical for clinical examination.

The electrooculogram (EOG) emerged as a clinically more feasible and readily implemented means of recording, and it has been used extensively since the 1970s. For recording horizontal saccades using an EOG, surface electrodes are placed at the bilateral canthi of the eyes, whereas for recording vertical saccades, electrodes are placed above and below one eye (Fig. 1). Unlike an electromyogram, an EOG is not meant to record the electrical activity of extraocular muscles. Rather, the EOG records changes in eye position based on the difference in electrical potential between the two surface 
A

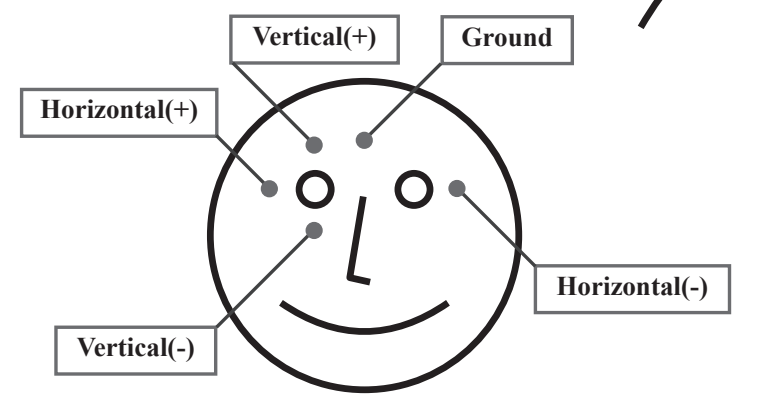

B

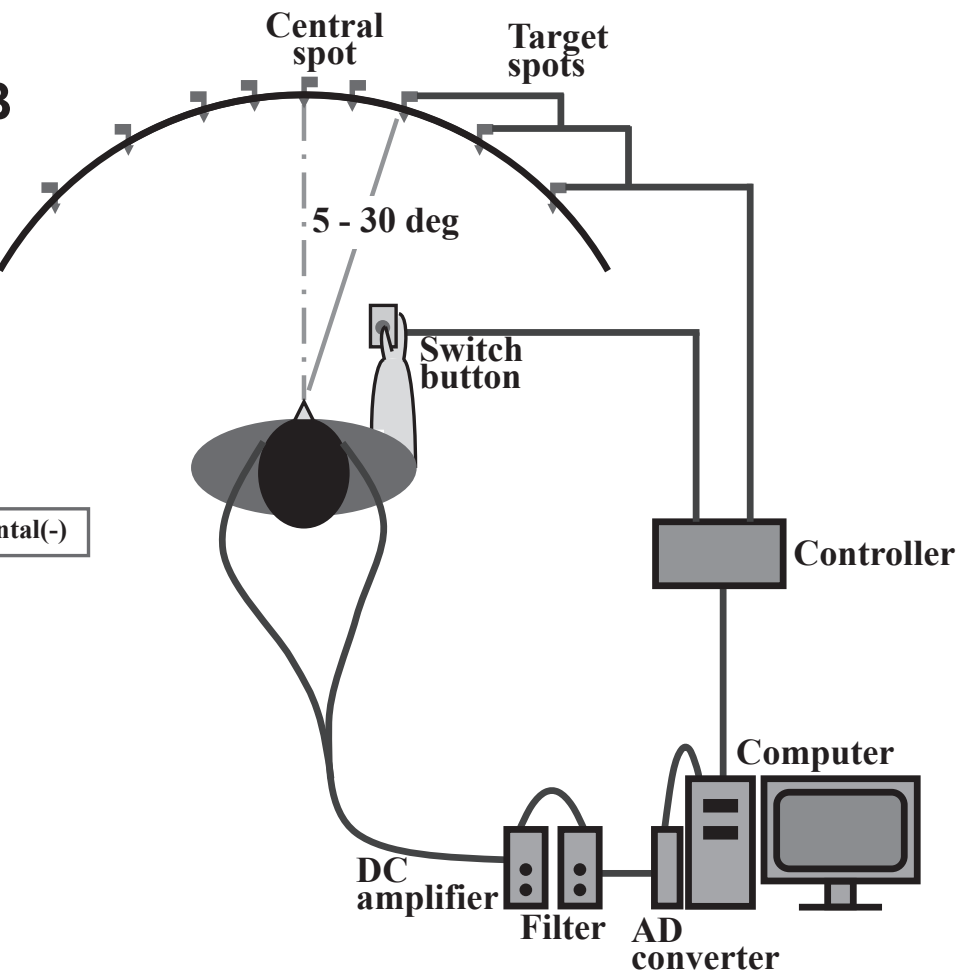

Fig. 1. Typical setup for recording an electrooculogram. A. For recording horizontal saccades, electrodes are placed at the bilateral outer canthi, whereas for recording vertical saccades, electrodes are placed above and below one eye. B. Subjects are seated in front of a black, concave, dome-shaped screen measuring $90 \mathrm{~cm}$ in diameter that contains light-emitting diodes embedded in pinholes, which serve as the fixation points and saccade targets. The subject holds a microswitch button connected to the microcomputer, allowing the subject to initiate and terminate a task trial by pressing and releasing the button. The target point is turned on at a random location $5,10,20$, or 30 degrees horizontally to the left or right of the central fixation point.

electrodes. Because the eyeball is positively charged in its anterior aspect, including the cornea, relative to its posterior aspect, the right electrode becomes more positive than the left when the subject turns his/her gaze to the right, and becomes negative when he/she gazes to the left. The potential difference between the electrodes is roughly linear to the angle of rotation of the eyes, up to 30 degrees $(5-30 \mathrm{deg})$, with a resolution of approximately 0.5 degrees. An EOG is an efficient method that can be implemented in a short time (several minutes) and has been widely used for patient studies. ${ }^{9)-11)}$ However, saccade measurement using an EOG may frequently be corrupted by artifacts, such as electromyography and electroencephalography signals, and the spatial resolution is not as good as with the video-based eye tracking method discussed later. Although an EOG is often considered outdated today, it correlates well with recordings obtained using video-based eye tracking systems for horizontal saccades. The drawback of this method is that it cannot accurately record vertical eye movements, because the correlation between the angle of rotation and the potential difference between the electrodes is not as good as for horizontal saccades. Consequently, recording in the vertical direction is more often used for monitoring blinks, rather than vertical eye movements.

At the present time, video-based eye tracking has become the standard method for clinical evaluation. In principle, this method is based on capturing an image of the cornea and calculating its center. ${ }^{12)}$ Infrared light is projected onto the cornea, and its point of reflection is also recorded by a chargecoupled device camera. Because the curvatures of the cornea and the eyeball differ slightly, the relative difference between these two positions can be used to measure the direction of the gaze. Subjects are first asked to look at several predetermined locations for calibration. The current direction of gaze is then recorded and calculated relative to the predetermined locations used for calibration. This method allows the examiner to record the direction of gaze, both 
horizontal and vertical, with a spatial resolution of up to $0.25-0.5$ degrees, and can thus be used for eye tracking studies (see last section). However, this method also has its drawbacks. For example, in Asian subjects with a narrow eye cleft, parts of the cornea are frequently covered by the eyelid, resulting in an inaccurate calculation of the center of the cornea. In addition, patients with cataract lenses, aberrant reflection of the infrared light may hamper recording of the gaze direction.

A third method for saccade recording is based on the use of a search coil, embedded in a modified contact lens and inserted into one eye, while the subjects' head is positioned in a graded magnetic field. When the eye with the search coil moves, it induces a current in the coil, which flows into the electric wire connected to it, ${ }^{13)}$ and this in turn can be used to measure the direction of gaze. Although this is the most precise method for recording eye movements, with a spatial resolution down to 0.1 degrees, this method is also painful and irritates the eye, if not more than the "suction cup" method of Yarbus. Thus, the recording can only be performed for 20-30 minutes at the most, even with the help of a topical anesthetic, and the hygiene of the cornea has to be maintained. In addition, recording can become inaccurate when the contact lens containing the search coil slips over the cornea.

\section{Two main classes of saccades}

Not all saccades have the same nature. Saccades are largely divided into two main classes; those that are made in response to the presentation of a visual target (externally guided saccades), and those made in the absence of a visual target (internally guided saccades). The two classes of saccades may appear superficially similar, but in reality, they differ greatly in terms of the brain processing required for their generation.

One of the most frequently used saccade paradigms is the visually guided saccade (VGS, Fig. 2A) task. In this oculomotor paradigm, the subjects are first required to fixate the central fixation spot. Some time after that, a target appears to the left or right of the central fixation point (or perhaps above, below, or even at an oblique angle) as the central fixation target is extinguished, and the subject is required to make a saccade toward that target. This type of task is called the reactive or externally triggered saccade task, because saccades are made in response to, or visually guided by, the appearance of a peripheral target.
An example of internally guided saccades made in the absence of a visual stimulus is the memoryguided saccade (MGS; Fig. 2B), either made alone or in a sequence. ${ }^{14)-19)}$ Similar to the above task, the subjects are first required to fixate the central fixation point as it appears. While the subject is gazing at this point, a cue appears for a brief period of time (e.g., for $50 \mathrm{~ms}$ ) in the periphery, typically to the left or right of the central fixation point, and is then extinguished quickly. The subject is required to remember the location of the presented cue, but not to immediately make a saccade towards it.

After a delay period of $1-2 \mathrm{sec}$, the central fixation spot is extinguished, upon which the subject is allowed to make a saccade to the remembered location of the target. Made in the absence of a visible target and guided by the memory of the cue location (hence the name MGS), this type of saccade is considered an internally guided saccade, which has a more voluntary nature than the VGSs discussed above. As we will explain in the next section, internally guided saccades are mediated by different neural structures than externally guided saccades, and have a slightly longer latency and slower peak velocity than the latter, when saccades of similar amplitude are compared. Therefore, it is useful to study both reactive (externally guided) and voluntary (internally guided) tasks in patients with neurological disorders. The contrast between the tasks allows a closer look into the pathophysiology underlying neurological disorders than if tasks are studied individually (see below).

Another saccade task frequently used in the clinical setting is the antisaccade (AS) task. ${ }^{20)-26)}$ The sequence of presentation of the central fixation spot and peripheral target is the same as in the VGS task, but in the AS task, subjects are required not to make a saccade toward the target as it appears, but toward the opposite direction (there may or may not be a gap period between the fixation offset and presentation of the target). This task has a more voluntary nature than the VGS task, in that the subjects have to suppress the prepotent tendency to make a saccade toward the presented target, but instead to make a saccade in the opposite direction, where no visual target is presented.

\section{Characteristics of normal saccades}

Figure 3 shows a typical trace of a saccade recorded using EOG in a normal subject. Time is shown along the x-axis, and eye position is shown along the y-axis. The trace deflects upwards or 


\section{A Visually guided saccade (VGS)}

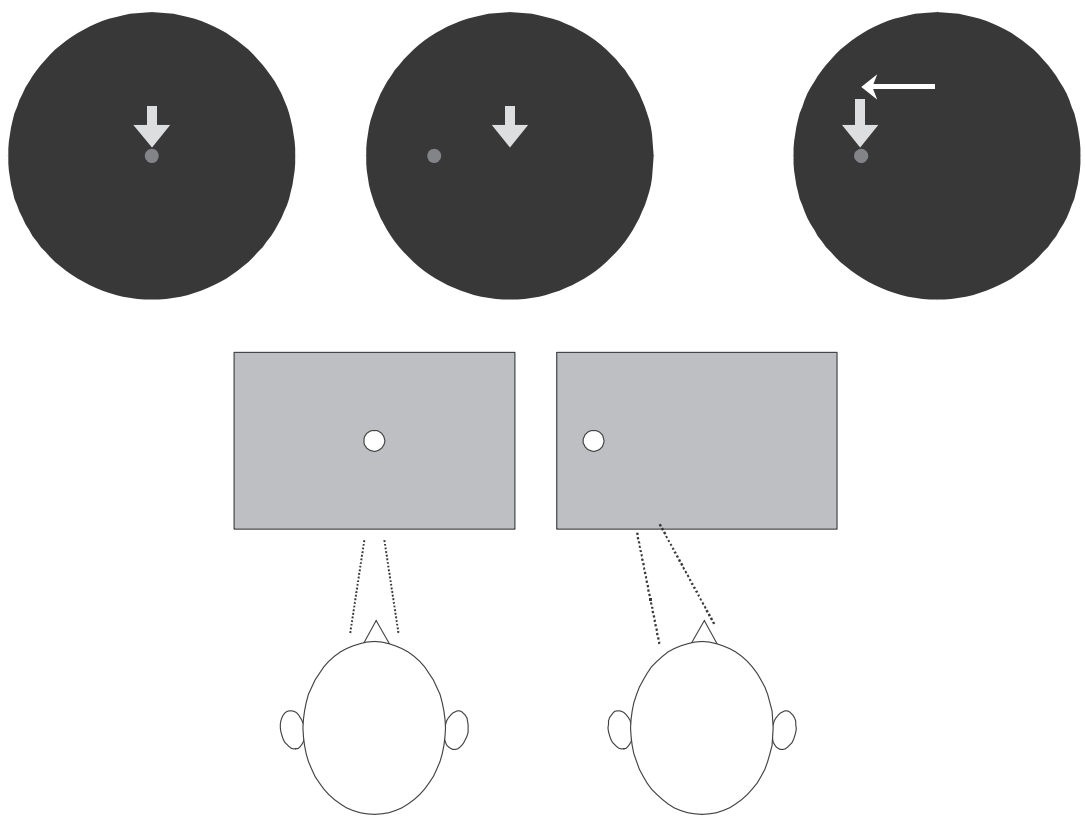

\section{B Memory-guided saccade (MGS)}
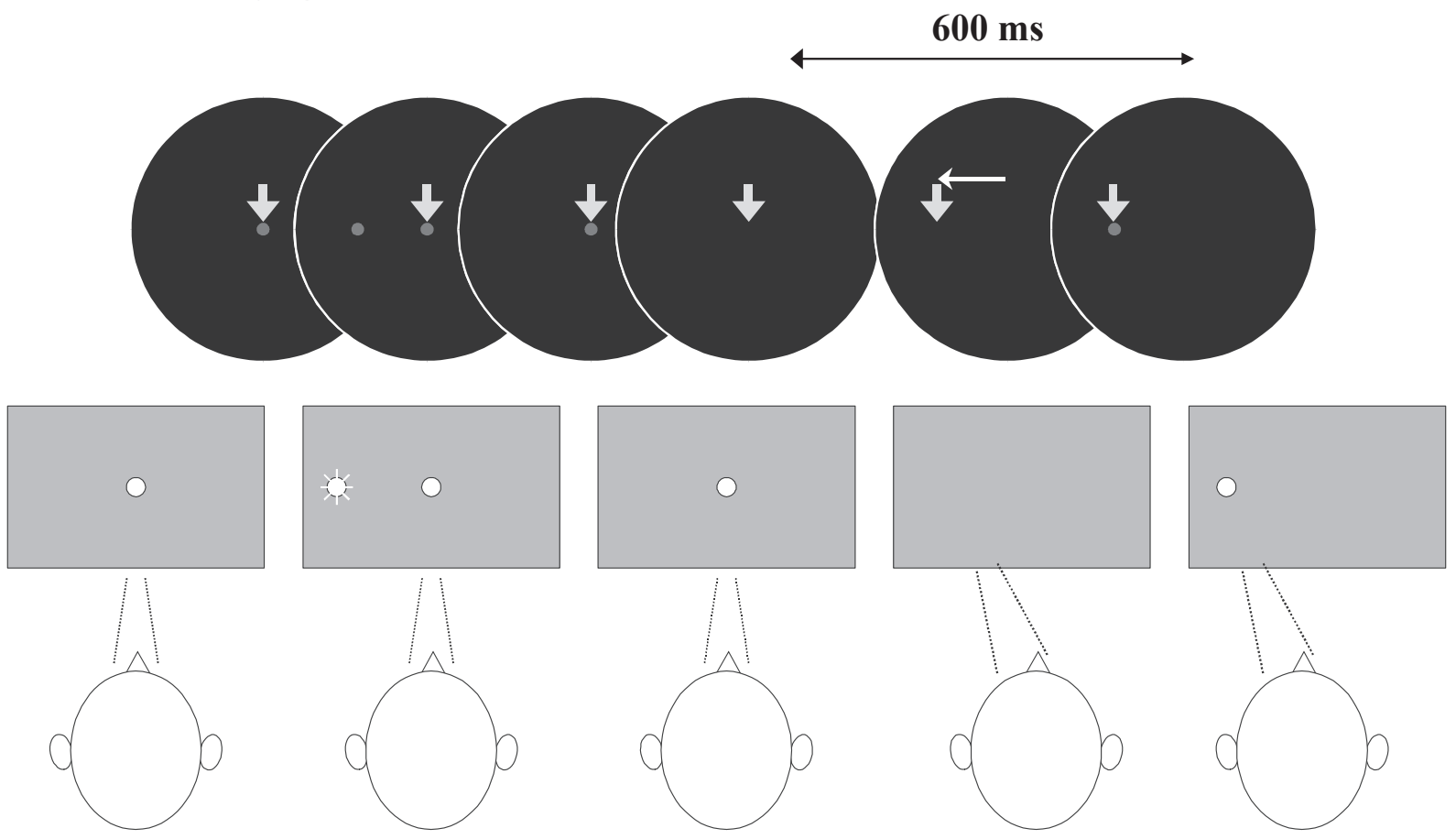

Fig. 2. Oculomotor tasks used in clinical saccade studies. A. Visually guided saccade (VGS), B. Memory-guided saccade (MGS). Reproduced with permission and modified from Terao et al. ${ }^{96)}$ 


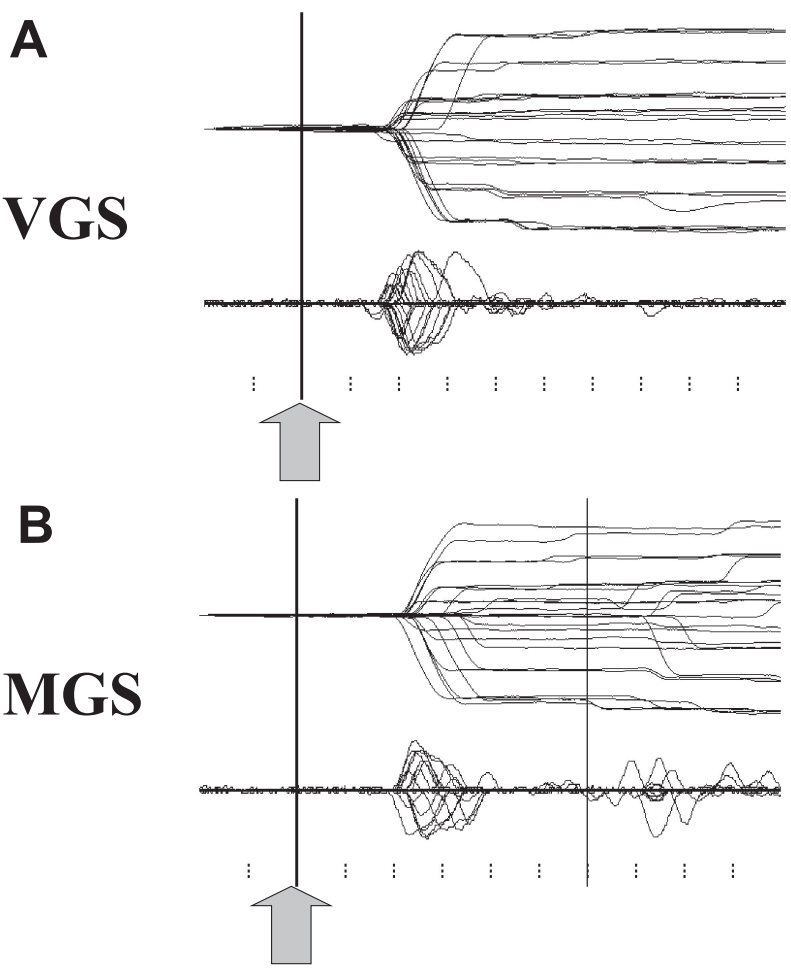

Fig. 3. Example of saccade records in a normal subject. A. Saccades records of visually guided saccade (VGS). B. Memoryguided saccade (MGS). A total of 25 VGS and MGS traces are superimposed, time-locked to the signal instructing the start of saccades, i.e., presentation of a target (VGS) or offset of the central fixation spot (MGS, shown by arrows and vertical bars). Lower traces in each figure depict the velocity profile of the saccades. The horizontal axis gives the time, and the vertical axis gives the eye position (upper trace) or velocity (lower traces). Ticks below are marked at 100-ms intervals.

downwards when a saccade is made toward the left or right. The vertical bar shows the time signaling the start of saccades. For VGSs (upper trace), this is marked by the presentation of the target, and for MGSs (lower trace), the time the central fixation point was turned off.

The first parameter to look at in saccade recordings is latency, which is measured from the time of target presentation (or extinction of the central fixation point) to the time when the deflection occurs. The step of the deflection gives the saccade amplitude, whereas the slope of the deflection corresponds to the speed of the saccade. The lower traces in each figure give the velocity of eye movements. The peak height of this velocity curve provides a measure of peak velocity.

Typically, the gaze can capture the target in just one step with remarkable precision; there is usually no need for a corrective saccade to adjust gaze position. Only when the target step is large (above 20 degrees or more of target eccentricity) will a single corrective saccade follow the first one.

In VGS tasks (upper trace), saccades are typically initiated $150-200 \mathrm{~ms}$ after target presentation. The velocity of the saccade, represented by the slope of the trace, is usually very fast, corresponding to a velocity of $200-500 \mathrm{deg} / \mathrm{sec}$ depending on saccade amplitude and type (see below). In MGS tasks (lower trace), the vertical bar shows the time when the central fixation point is extinguished and subjects are allowed to move their eyes (see Two main classes of saccades section above). These saccades occur at a latency of $200-250 \mathrm{~ms}$, thus slightly later than VGSs. The slope of the deflection, i.e., saccade velocity, is also slightly slower than for VGSs. Again, the target is captured in one saccade step.

As noted above, this accuracy in capturing the target is one of the spectacular features of the saccade system, with which virtually any normal subject is endowed; fast as they are, saccades in normal subjects can precisely capture the target, usually in a single step. This is a remarkable feat, comparable to throwing a ball to hit a distant target. In most cases, only a professional baseball player would be able to achieve this. Not only are saccades fast, but they also have a short duration of several tens of milliseconds, which implies that no feedback can reliably correct their amplitude during execution, and that the movements have to be preprogrammed by the brain. Once saccades are initiated, correction, if necessary, is made on-line only by feedforward rather than feedback control, which is the role of the cerebellum (see below). Achieving this level of accuracy despite the short duration and high speed of saccades requires an exquisitely dedicated control system, which we will discuss in the next section.

Accuracy and speed are not the only remarkable features of gaze movements. Although we can move our gaze quickly and immediately when necessary, we can also suppress eye movements depending on the context; apparently, humans do not "reflexively" respond to and look at every human or object of interest. Thus, gaze control in higher animals is not only a matter of saccade generation with accuracy and speed, but also of suppression.

\section{Which cortical regions are responsible for initiating gaze movements?}

For studying saccade performance in humans, it is important to know what function each neural 

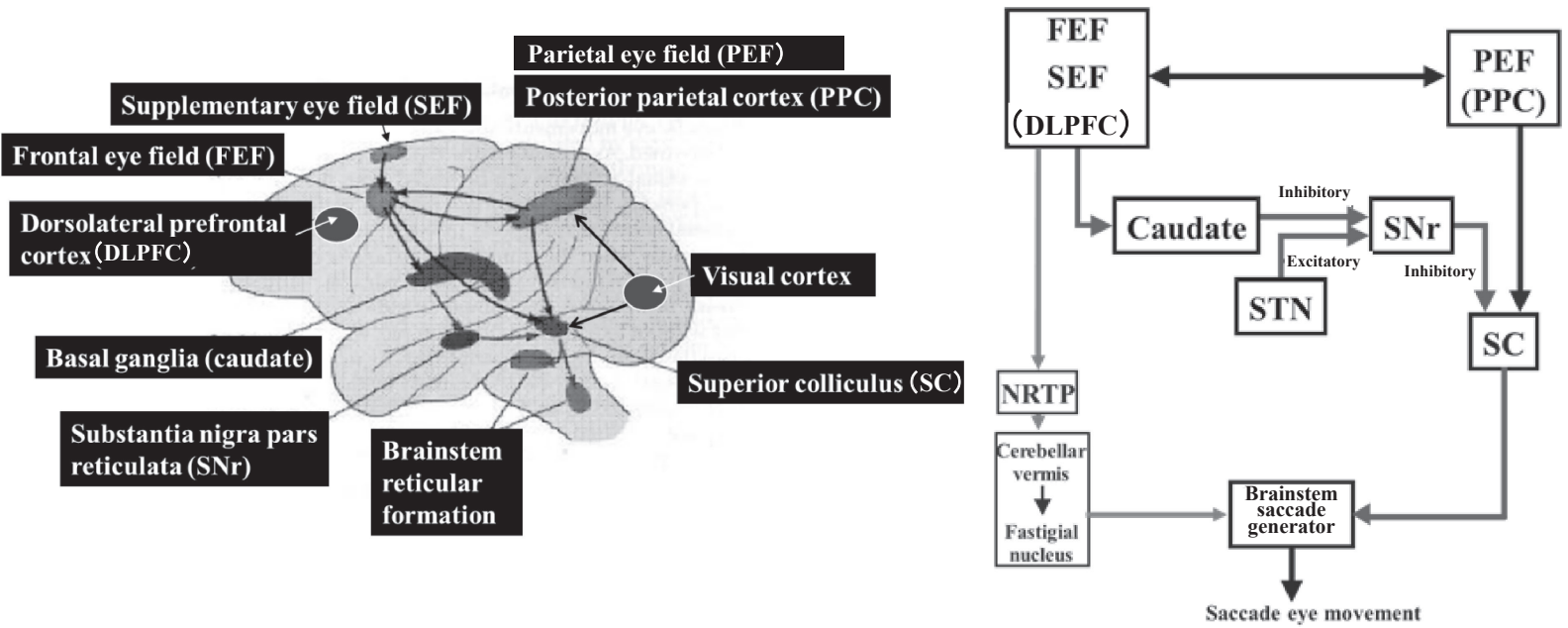

Fig. 4. A. Main neural structures for controlling saccades. B. Schematic diagram depicting the involvement of the basal ganglia and cerebellum in saccade generation. DLPFC: dorsolateral prefrontal cortex, FEF: frontal eye field, NRTP: nucleus reticularis tegmenti pontis, PEF: parietal eye field, PPC: posterior parietal cortex, SC: superior colliculus, SEF: supplementary eye field, SNr: substantia nigra pars reticulata, STN: subthalamic nucleus.

structure serves for generating and inhibiting saccades at the systems level. Before reviewing our research to demonstrate how saccade studies can be used to investigate the pathophysiology of patients with neurological disorders, we will present a brief overview of the oculomotor (saccade) system, as shown in non-human primate studies.

Commands to initiate voluntary saccades arise at the cortical level and are sent to subcortical levels and the brainstem, which contain saccade generators. Broadly, three main cortical areas contribute to the control of saccades: the frontal eye field (FEF), the supplementary eye field (SEF), and the parietal eye field (PEF, or posterior parietal cortex $[\mathrm{PPC}]$; Fig. 4A; see $\mathrm{Munoz}^{17)}$ for review), the projections of which converge into the superior colliculus (SC).

The FEF and PPC are especially important for initiating saccades, and in primates, saccades can be elicited from these areas using electrical stimulation. Single neurons of the FEF discharge before saccades mainly toward the contralateral side, and saccadic eye movements are evoked by microstimulation at the sites of these neurons. In the FEF, a somatotopic organization of the saccade is observed at different sites. The direction of the saccades evoked at each site changes systematically with small advances of the stimulating microelectrode, and all contralateral saccadic directions are represented in a single electrode penetration down the bank of the arcuate sulcus. ${ }^{27), 28)}$ Neural activity that precedes the initiation of an eye movement has also been recorded from the PPC (lateral intraparietal cortex [LIP]), ${ }^{29), 30)}$ and electrical stimulation of the parietal cortex, both within the LIP and in surrounding regions, can evoke saccadic eye movements. ${ }^{29)-34)}$ Similarly, saccades can be evoked from electrical stimulation of the SEF at the medial aspect over the lateral convexity of the frontal lobe. ${ }^{35)-37)}$ The human counterpart to the $\mathrm{SEF}$, located anterior to the supplementary motor area, also projects to the SC. $^{24)}$

The descending pathways from different oculomotor cortical regions are thought to serve different roles. Generally speaking, the parietal cortex plays a more important role in triggering automatic, or reactive (reflexive), saccades, ${ }^{38}$ ) whereas for more deliberate or cognitively demanding oculomotor tasks, the frontal lobe, including the FEF, plays a greater role. This has also been shown in human neuroimaging studies. ${ }^{39), 40)}$ The SEF appears to be involved in more complex, cognitively demanding eye movements, e.g., in the control of more complex saccades sequences, or saccades combined with body movements. ${ }^{37), 41)}$ From a functional point of view, the fronto-basal ganglia-SC pathway may also be important in selecting the target and specifying the timing of volitional saccades, whereas the parietotectal pathway appears to be important in determining their metrics (see Andersen et al. ${ }^{42}$ ) for review). 
Supranuclear saccade commands from these cortical areas (the FEF, PPC, and SEF) arrive and converge in the SC. The SC then sends its output to the saccade generators in the brainstem reticular formation, namely the paramedian pontine reticular formation (PPRF) in the pons for horizontal saccades, and the rostral interstitial nucleus of the medial longitudinal fasciculus (riMLF) in the midbrain for vertical saccades. Finally, the brainstem saccade generator sends its output to the motoneurons (MNs) of the oculomotor nuclei, which directly innervate the external oculomotor muscles. Specifically, excitatory burst neurons (EBNs) and inhibitory burst neurons (IBNs) in the brainstem reticular formation are silent during fixation and discharge bursts of action potentials for saccades in the ondirection during saccade generation (for review see Munoz ${ }^{17)}$ ). The EBNs excite the on-direction MNs monosynaptically, and also excite IBNs on the contralateral side. The IBNs in turn inhibit the antagonist MNs. In this way, the $\mathrm{SC}$ receives supranuclear saccade commands and is involved in target selection as well as saccade initiation by providing a trigger signal mainly to the brainstem saccade generator, comprising the "final common pathway" for voluntary saccades (see Hikosaka et al., 2000 for review). Because saccades are so brief that there is not enough time for visual feedback to guide them to the target, the brain must specify the command for a saccade before it starts. ${ }^{43}$ ) Thus, it may be possible to see the functional changes of these brain regions from the saccade record, i.e., how the brain pre-programs saccades.

The frontal and parietal oculomotor cortical regions are also known to have dense reciprocal connections. The model of Brown ${ }^{44)}$ posits that visual inputs to the SC dominate "reactive" saccade movements by default and allow fast, "reactive" saccades, such as VGSs, to take place toward a suddenly appearing target. When a planned movement is required instead, frontal activity can suppress reflexive responses to targets and allow time for processing a planned response to a different target. The interaction between the frontal and parietal cortices is important for this switch to occur, and may be disrupted by parietal lesions. In other words, the parietal lobe sets the stage for making a saccade toward a certain location, by activating the corresponding representation of possible target locations within the SC, whereas the frontal lobe plays a more important role in saccade triggering for executing voluntary saccades toward the selected target, or planning for a more cognitively demanding type of saccade. Consistent with this view, human neuroimaging studies have also shown that the PPC is more active in reactive saccades, whereas the involvement of frontal cortical regions becomes more evident for more cognitively demanding tasks, such as MGS and AS. ${ }^{34), 37,39)-41), 45)-47 \text { ) }}$

To assess saccade initiation in oculomotor tasks, we look at latency as a measure of saccade initiation. Saccade latency mainly reflects the time required by cortical processes, such as target selection and decision making. Saccade latency is prolonged when the neural structures involved in triggering saccades are affected. This has been studied in humans by investigating saccade performance in patients with focal brain lesions, especially cortical lesions. Studies have shown that several cortical areas contribute to saccade generation, both to their initiation and for calculating saccade metrics (see Gaymard et al. ${ }^{48)}$ for review; also see discussion of Fig. 13A below). Although the latency changes associated with focal lesions are relatively modest, parietal lesions including the PPC are associated with prolonged latency of reactive saccades, such as VGSs, whereas lesions in both the FEF and PEF are associated with prolonged latency of voluntary saccades, such as MGSs. ${ }^{49)-51)}$ Saccade latency is also prolonged with lesions of the anterior and posterior limb of the internal capsule for MGSs and VGSs, respectively. ${ }^{52)}$ Therefore, saccade latency of reactive saccades, such as VGSs, is mainly determined by the cortical commands from the PEF to the SC through the projection traveling through the posterior limb of the internal capsule. ${ }^{51)-54)} \mathrm{By}$ contrast, the latency of MGSs, a cognitively more demanding oculomotor paradigm, is determined by the cortical commands for saccades travelling from the FEF and PEF to the SC. ${ }^{51), 55)-57)}$ The former pathway projects to the SC through the anterior limb of the internal capsule. ${ }^{51), 55)-57)}$

\section{The role of the basal ganglia and the cerebellum} in saccade control

The basal ganglia (BG) and the cerebellum serve as side loops to this final common output pathway from the SC downwards, modulating the neural system for saccade generation. Once again, it is important to note that the SC serves as a nodal point for all kinds of voluntary saccades. The frontal lobe projects to the SC, either directly or indirectly through the BG, and it is through this latter pathway that the BG modulate the saccade system (Fig. 4B). Hikosaka et al. were the first to show that one of the 


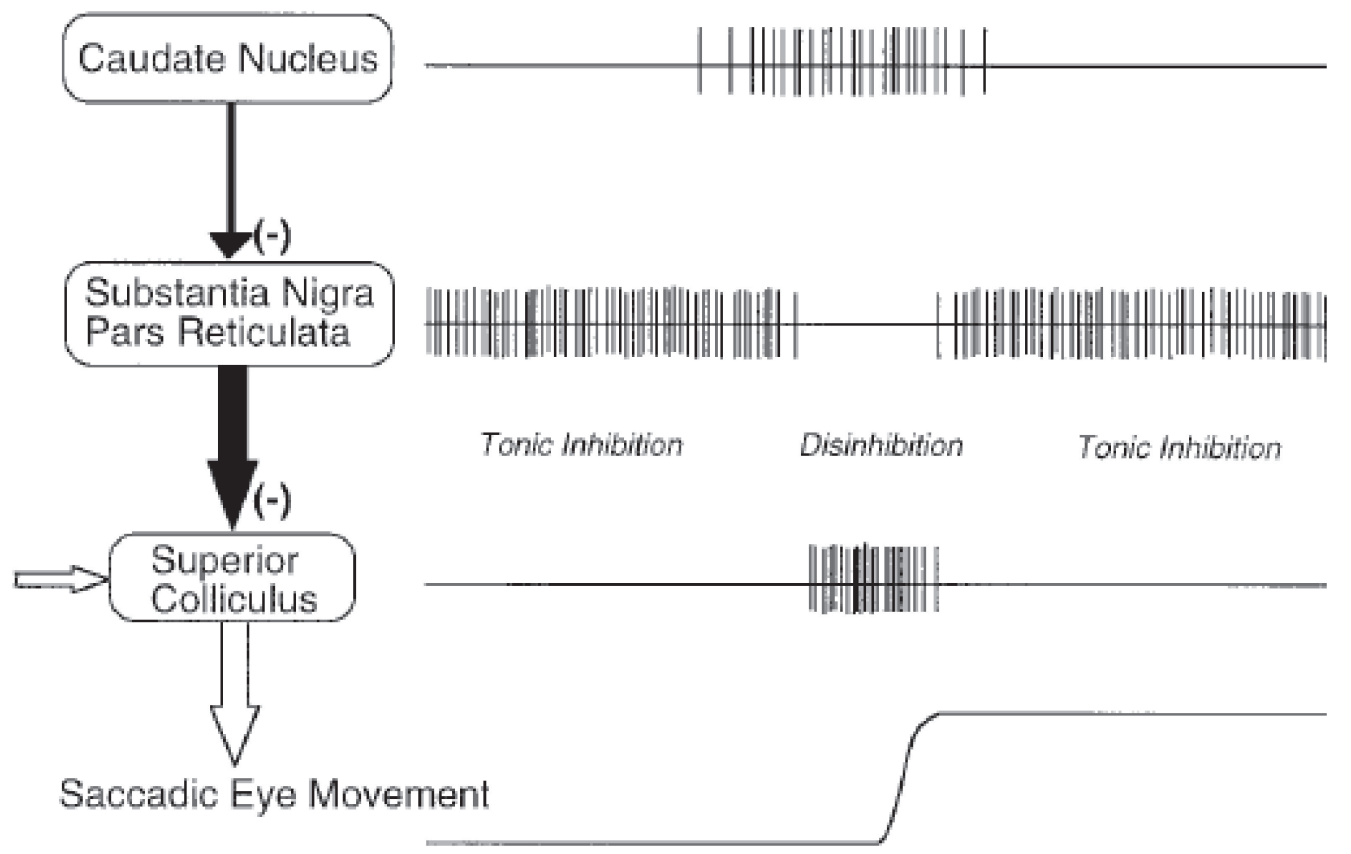

Fig. 5. Neural mechanism for generating memory-guided saccades (MGSs). Hikosaka et al. showed that the initiation of an MGS is mediated by cortical commands projected via the caudate nucleus and substantia nigra pars reticulata (SNr) to the superior colliculus (SC). This pathway corresponds to the direct pathway of the basal ganglia (BG). It contains two inhibitory neurons, i.e., from the caudate to the SNr, and then from the SNr to the SC, hence the name double inhibition pathway. A phasic inhibition of the highfrequency firing of the SNr by the caudate nucleus disinhibits the downstream SC, allowing a saccade to occur. Reproduced with permission from Hikosaka et al. (2000). ${ }^{58)}$

important functions of the BG is the generation of voluntary saccades, which are mediated by the BG circuit, comprising two successive inhibitory neurons; the BG (caudate nucleus) send inhibitory projections to the substantia nigra pars reticulata $(\mathrm{SNr})$, which in turn sends inhibitory inputs to the saccade neurons in the SC (Fig. 5). ${ }^{47)}$ At rest, the caudate neurons fire at a low intensity, whereas SNr neurons, downstream from it, are disinhibited and fire at a high frequency, thereby suppressing SC saccade neurons further downstream. When one intends to make a saccade, input from the cortex arrives at the caudate nucleus, temporarily resulting in enhanced activity of the caudate neurons. The activity of the $\mathrm{SNr}$ is then transiently suppressed, which in turn disinhibits the downstream SC, allowing saccades to occur.

Another important pathway modulating the saccade system is that involving the cerebellum (Fig. 4B). The pontine nuclei (the NRTP) receive cortical commands and project to the cerebellum and brainstem reticular formation. The output signals of the cerebellum are then emitted from the Purkinje cells of the dorsal vermis to the pontine burst and omnipause neurons by way of the cerebellar fastigial nuclei $(\mathrm{CFN})$, which in turn modulate the duration and amplitude of saccades by projecting back to the brainstem saccade generator to terminate directly on the EBNs and IBNs in the brainstem.

This output pathway plays an important role in generating saccades that land accurately on the target position by adjusting the saccade trajectory ${ }^{59), 60)}$ using accelerating and decelerating signals sent to the brainstem saccade generators. Purkinje cells of the dorsal vermis play an essential role in adjusting the duration of saccades online and maintaining their accuracy. In this process, the cerebellar output has been shown to accelerate contralateral saccades early in the movement, and to decelerate ipsilateral saccades later in the saccade, although the two signals actually show a substantial temporal overlap. ${ }^{61-65)}$ The early phase of a saccade, especially the accelerating phase as well as the peak velocity, may reflect the accelerating function of the cerebellum, whereas the late phase of a saccade, especially its deceleration phase, may reflect the braking function of the cerebellum. ${ }^{66)}$ Thus, when an initiated saccade happens to start with a velocity that results in a larger amplitude than was intended, the cerebellum suppresses the saccade-generating pathway in the brainstem and decreases the amplitude. Conversely, 
when the saccade amplitude is smaller than intended, the cerebellum accelerates and increases the amplitude more than was pre-programmed. Bilateral functional blocking of the CFN in monkeys reduces both saccade acceleration and deceleration, ${ }^{67)}$ resulting in a slightly decreased peak velocity (defective accelerator function), along with a significantly longer saccade duration, especially in its decelerating phase (defective braking function), than without functional blocking (also see the Neural structures implicated in determining saccade metrics section).

\section{What brain regions are responsible for inhibitory control of gaze?}

Another function of the saccade system with equal importance is the inhibitory control of unwanted (reactive) saccades, which, depending on the context, allows us to initiate a saccade when it is required, and to suppress it when inappropriate.

How can we study the function of inhibitory control? One of the tasks that can be used is the AS task. In this task, subjects are required to perform a saccade not toward the presented target, but instead to the position mirror-symmetrical to the central fixation point. Thus, this task requires the ability to suppress a reactive saccade toward the target; when this suppression fails, the subjects will perform a saccade (termed prosaccade) toward the presented target location. The occurrence of prosaccades indicates impairment of inhibitory control (Fig. 6A).

The MGS task can also be used to study the inhibitory control of reactive saccades. In this task, the cue is presented for a brief period to indicate the location of the future target, but subjects are required not to make a reactive saccade towards the cue when it appears. If the subject does make a saccade (termed saccade to cue), it means that he/she was unable to suppress the reflexive saccade toward the cue, i.e., the inhibitory control of reactive saccades is impaired (Fig. 6B); an increase in the frequency of saccades to cue also indicates impaired suppression of unwanted saccades.

Several cortical and subcortical structures have been implicated in the voluntary suppression of saccades. Pathways that exert inhibitory effects on the saccade system include the direct inhibitory projection of the FEF to the SC, altered BG output from the SNr, actions of omnipause neurons of the midline pontine reticular formation, the fixation cells of the SC, and omnipause neurons and IBNs of the brainstem. Receiving convergent input from almost all of the cortical and subcortical structures, the SC

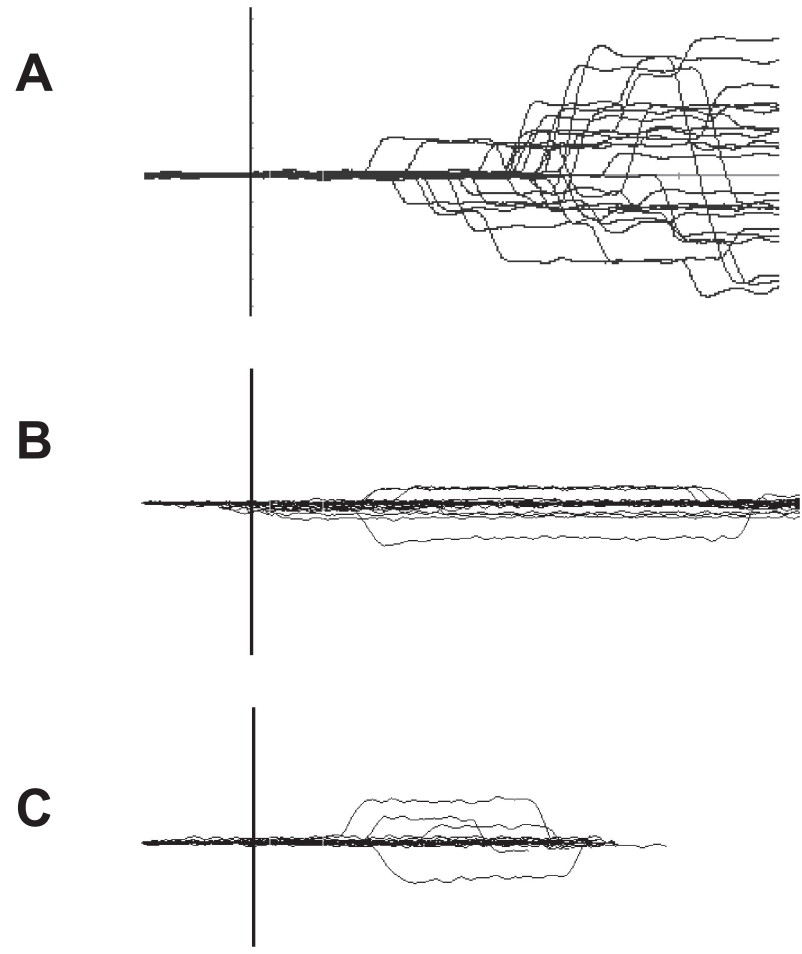

Fig. 6. An example of impaired inhibitory control of gaze. A. Prosaccades in the antisaccade (AS) task. Traces are aligned to the time of target presentation. In this task, the subjects are asked to make a saccade in the direction opposite from the target, but in some trials, subjects make inadvertent saccades, termed prosaccades, towards the target as shown in this figure. After making prosaccades, subjects usually make corrective saccades in the instructed direction, i.e., opposite from the target. B. Saccades to cue in the memory guided saccade (MGS) task. Although this subject was asked to keep fixating the central fixation point when a peripheral cue appeared for a brief period (time of appearance is shown by the vertical bar), inadvertent saccades (saccades to cue) were made in some trials. C. Saccades to target in the reaction time task. In this task, the subjects are asked to release the button as soon as the peripheral visual spot comes on (time of appearance is shown by the vertical bar), while keeping the central fixation point fixated. This subject made inadvertent saccades (termed saccades to target) in some trials.

forms a vital node, not only in the generation of voluntary saccades, but also in voluntary fixation and suppression of unwanted saccades. The main inhibitory inputs arriving at the SC come from the frontal lobe and BG. Inhibitory control of saccades is thus impacted when focal lesions affect the frontal lobe and caudate nucleus. ${ }^{51), 55)-57), 68), 69)}$

As discussed above, MGSs also use this pathway of inhibitory control for saccade generation (see Hikosaka et al. ${ }^{14)}$ for review). The SNr fires at a high frequency in the resting state, and suppresses saccade neurons of the downstream SC by default. However, 
when a saccade is required and the saccade command arrives from the cortex via the caudate nucleus, the $\mathrm{SNr}$ is temporarily suppressed, and disinhibits the SC downstream from it, allowing saccades to occur. It appears that for the saccade system, holding the gaze still using these inhibitory pathways is the "default mode". Only when gaze movements are required, is the oculomotor system temporarily released from suppression, i.e., from the "braking" signals, to allow saccades to be initiated. Once again within a short timeframe, the saccade system returns to its "default mode", and the gaze is stopped under the control of the "brake".

For inhibiting prosaccades in the AS task, the pathway from the frontal lobe that projects directly to the $\mathrm{SC}$ is thought to play an important role; lesions in the dorsolateral prefrontal cortex (DLPFC) reduce the ability of subjects to suppress reflexive prosaccades in the AS task. ${ }^{68), 69)}$ Tinsley and Everling $^{70)}$ described a role for neurons in the DLPFC in fixation control; the authors demonstrated that many neurons in the DLPFC show discharges that are modulated by the gap period in the gap saccade (GS) task. In the GS task, the fixation point is turned off for some time (the gap period) before target presentation.

The parietal lobe has also been implicated in the inhibitory control of saccades. In primates, PEF neurons show modulation similar to that described in the DLPFC for inhibitory control. ${ }^{71), 72)}$ In humans, parietal lesions are also associated with an increased frequency of saccades to cue. ${ }^{51), 73)}$

\section{Neural structures implicated in determining saccade metrics}

The oculomotor system is not only important for triggering or suppressing saccades, but also for determining saccade metrics, i.e., for performing a saccade with the required amplitude and velocity.

One of the main structures implicated in saccade metrics is the PPC. The PPC is engaged in the sensorimotor integration required for generating a saccade. The PPC computes target position based on both visual stimuli (VGSs, GSs) and memory of target position, and this information is sent to the $\mathrm{SC}$, setting the stage for saccade initiation as described above. ${ }^{74)-76)}$ In the PPC, saccade metrics are pre-programmed before a saccade is launched, as compared with the cerebellum, which controls saccade metrics online. Additionally in humans, the PPC plays an important role in computing target position, based on both visual stimuli (VGSs, GSs) and memory of target position. ${ }^{53), 54), 77)}$ In fact, although oculomotor impairment after focal damage of the PPC is relatively modest, decreased saccade accuracy, typically hypometria, has also been observed in humans after lesions in the pathway from the PPC to the SC. ${ }^{51)-53)}$

By contrast, the cerebellum plays an important role in the online control of saccades. Unlike the frontal-parietal cortex-SC projection, this second pathway involving the cerebellum has generally been thought to play only a minor role in saccade initiation. ${ }^{30), 31)}$ However, this pathway is important for performing an accurate saccade by enabling the gaze to arrive at a desired position in a single step (saccade). ${ }^{6), 32)}$ As discussed above, this is achieved by the cerebellar output nuclei sending accelerating and decelerating signals to the brainstem saccade generators. ${ }^{67)-70)}$ Cerebellar patients whose gaze control is compromised cannot stop a saccade exactly where they want, because the velocity fails to reach a sufficient level due to reduced acceleration, and/or the braking effect fails to stop the action at the appropriate time. ${ }^{6), 51), 78)-85 \text { ) }}$

\section{What are the main saccade abnormalities in neurological disorders, and what is their relationship to pathophysiology?}

Recent advances in neuroimaging studies have provided detailed information about the function of brain networks associated with the performance of oculomotor tasks at the systems level. However, direct application of neuroimaging to clinical studies is difficult, not only because the necessary setup is not available in all hospitals, but also because the activities of relevant cortical and subcortical regions may be differentially increased or decreased in patients relative to normal subjects, e.g., as a result of functional compensation, so that the interpretation of results becomes difficult. In this respect, saccade recording is very useful if the brain dysfunction can be discerned from the pattern of abnormality in the saccade records, specific to each neurological disorder, such as cerebellar ataxia and parkinsonism. With the extensive neuroanatomical and neurophysiological knowledge already obtained from non-human primate studies, we should be able to obtain information about the brain dysfunction of neurological patients from the saccade records. ${ }^{6), 7), 86), 87)}$ Clinically, this is especially helpful for neurological disorders in children, in whom, despite apparent clinical symptoms exhibited by these patients, magnetic resonance imaging and computed tomography frequently do 
VGS

Normal

Early PD
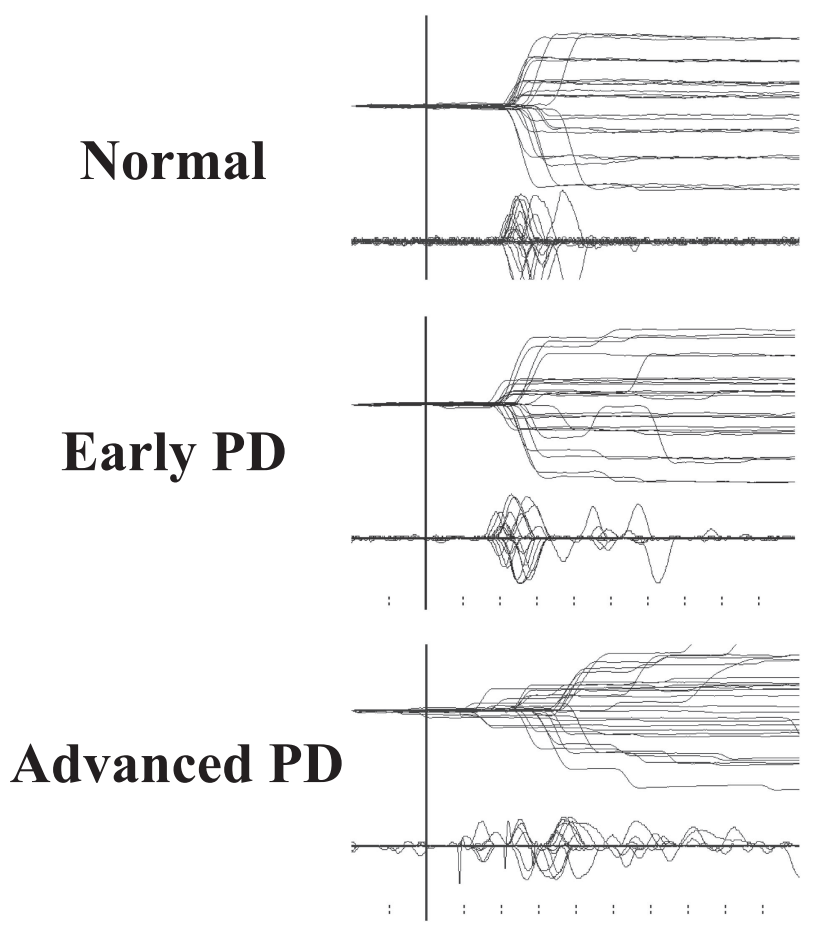

MGS
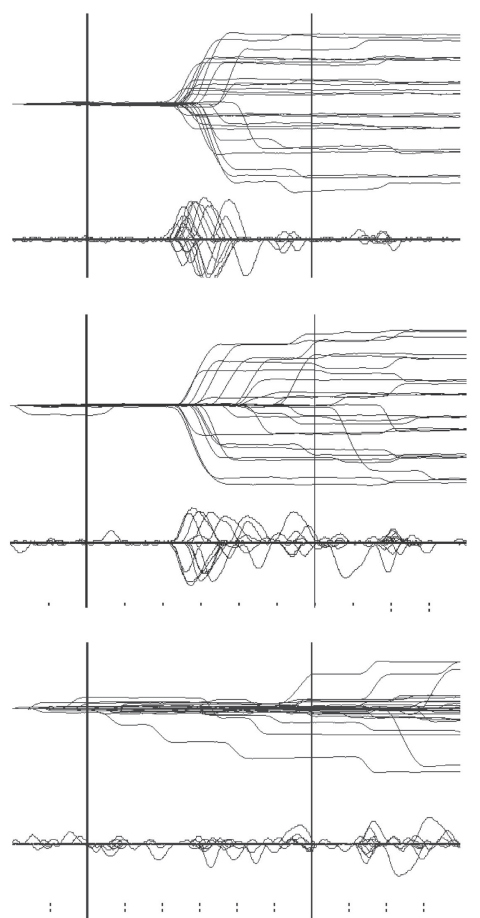

Fig. 7. Saccade records in patients with Parkinson's disease (PD). Saccade records of patients with early (middle traces) and advanced $\mathrm{PD}$ (bottom traces) are shown in comparison with those of a normal subject (top traces). Conventions as in Fig. 3. Superimposition of traces for 20-30 trials each. The left half shows a visually guided saccade (VGS), the right half shows memory-guided saccade (MGS). Saccades are characterized by hypometria in both tasks. In addition, MGSs are more affected than VGSs in that the latency is more variable and that failed trials also predominate at an advanced stage. Reproduced with permission from Terao et al. ${ }^{10)}$

not reveal anything about the localization of lesions within the brain.

Saccade recording can be implemented within a relatively short time and quite non-invasively. Unlike more complex cognitive tasks, simple oculomotor tasks can be adequately performed by patients with neurological disorders, even with compromised cognitive function. In addition, although damage to the corticospinal tract frequently leads to complete paralysis with no movements of the contralateral limbs, almost all patients exhibit some level of saccade activity that can be recorded, even in the presence of cortical and subcortical lesions affecting oculomotor control. This may be because saccade eye movements are not controlled by discrete regions serving clearly discrete functions, but by partially redundant systems performing their role in integrated, widely distributed brain circuitry, as discussed above.

We have recorded saccade performance in over 250 patients with neurological disorders, and in about 400 normal, age-matched control subjects.
In the following sections, we will describe typical patterns of eye movement records in major neurological disorders, and examine what saccade recording can tell us about the dysfunction of neural structures related to saccade control and the pathophysiology underlying the disorders.

a) Parkinsonism: BG pattern of saccade abnormality. Saccade performance has been frequently studied in patients with Parkinson's disease (PD), a progressive neurodegenerative disorder characterized by BG dysfunction. Because the pathological brain areas in the oculomotor system in the earliest stages of the disease are typically restricted to the substantia nigra pars compacta, whose dopamine neurons project to the striatum, $\mathrm{PD}$ provides insights into how BG dysfunction affects saccade performance.

In the example shown in Fig. 7, VGS and MGS performances in early and advanced PD patients are contrasted with those in a normal subject (top trace). In these traces, it is apparent that in $\mathrm{PD}$, the MGS 
performance was lower than the VGS performance. MGS latency is prolonged in patients with PD, compared with normal subjects, whereas VGS latency is relatively well preserved, with similar latencies as in normal subjects. The prolongation of MGS latency becomes more prominent with progressing disease, such that patients at advanced stages even fail to initiate a saccade in some trials. By contrast, both VGSs and MGSs show small saccade amplitudes (hypometria), with the saccade reaching the target location with a sequence of multiple saccades, although the magnitude of hypometria is again much greater for MGSs. The magnitude of hypometria increases with disease progression.

As mentioned above, the amplitude of saccades in PD patients is smaller than normal, so that the gaze does not reach the target in one step, but instead requires a sequence of multiple, corrective saccades. $^{88)-96)}$ In PD patients, hypometria is universally observed in oculomotor paradigms. ${ }^{88)-96)}$ Although parkinsonism is one of the most common causes of hypometria, it can also be seen in other neurological disorders, including cerebellar ataxia.

How does this pattern of saccade abnormality in $\mathrm{PD}$ patients come about, even though these patients have no focal lesions in the frontal and parietal oculomotor cortical regions? Saccade latency may be affected by the excitability of the SC, which comprises the final common pathway for voluntary saccades. Pathophysiologically, SC activity in $\mathrm{PD}$ is excessively inhibited by the output from the BG, mediated through the $\mathrm{SNr}$ to suppress the excitability of the $\mathrm{SC}^{95), 96)}$ (Fig. 7), leading to hypometria. Non-human primate studies have shown that electrical stimulation of the $\mathrm{SNr}$ that inhibits the downstream SC reduces the amplitudes of both VGSs and MGSs. ${ }^{97)}$ Conversely, increased activity of the SC is associated with a shorter saccade latency. ${ }^{98)}$ As discussed above, the BG pathway is more involved in voluntary saccade tasks made in the absence of a visual target than in generating reactive saccades made in response to a visual target. Thus, voluntary saccade paradigms, such as MGSs, are more affected in PD patients, whereas reactive saccade tasks, such as VGSs, are relatively spared.

Although characteristically observed in patients with parkinsonism, hypometria is not pathognomonic of parkinsonism, but may also be seen in various other neurological conditions, such as cerebellar disorders (see below). In these cases, the feedforward adjustments of saccade amplitude and trajectory by the cerebellum are compromised; the saccade pro- gram does not match the intended saccade output, such as saccade amplitude, to capture the target. This can also be seen in normal subjects, especially in VGSs made with a short latency; there is a latencyamplitude trade-off for VGSs, where smaller saccades can be made more "automatically" with a shorter latency than large-amplitude saccades. ${ }^{99)}$ Larger saccades to the same target are made with a slightly longer latency.

Inhibitory control of saccades is also affected in PD (Fig. 8), with increased occurrence of saccades to cue. ${ }^{100)-108)}$ This abnormality may be explained by the excessive but leaky output (impaired suppression) of the BG suppressing the output stage of volitional saccades, the SC, and the downstream brainstem structures. ${ }^{96)}$

b) Cerebellar ataxia: cerebellar pattern of saccade abnormality. Figure 9 shows a typical example of a saccade recording in a patient with spinocerebellar degeneration (SCD). The main neurological manifestation is ataxia, attributed to cerebellar dysfunction, which is characterized by a lack of voluntary coordination of muscle movements.

At first glance, a marked variation in saccade amplitudes can be noted across trials, especially in patients at advanced stages; in some trials, saccades pass beyond the location of the target (hypermetria), but in others, they fall short of it (hypometria), so that corrective saccades are made in both cases to adjust gaze position. ${ }^{109)-112)}$ A closer look at the traces reveals hypometric VGS in most trials, while MGS can be hypermetric as well as hypometric. ${ }^{113), 114)}$ However, noting that while hypermetric VGSs and MGSs have frequently been associated with cerebellar disorders, they are not specific to cerebellar ataxia and can also be seen in other neurological disorders, including parkinsonism. Hypermetria can even occur in normal subjects, in whom saccades made with a short latency in the MGS task may often be hypermetric.

The velocity profiles of saccades (Fig. 10) can also tell us how the braking and accelerating signals from the cerebellum are affected. SCD patients in whom cerebellofugal pathways are affected present with velocity profiles showing decreased peak velocity due to impaired acceleration and longer decelerating periods (Fig. 11). ${ }^{81)}$ These velocity profiles are similar to those seen in primates with functionally blocked CFN on both sides, which results in decreased saccade acceleration and deceleration. ${ }^{115), 116)}$ The variable amplitude across trials can be explained by the impaired braking and accelerating function by 
Saccades to cue

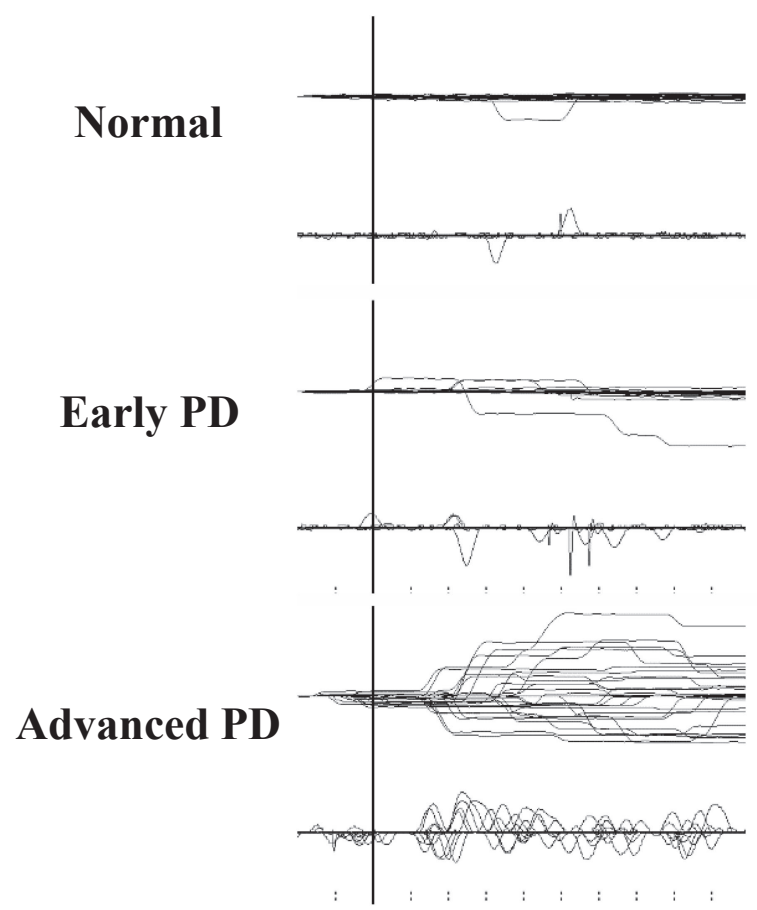

Saccades to target

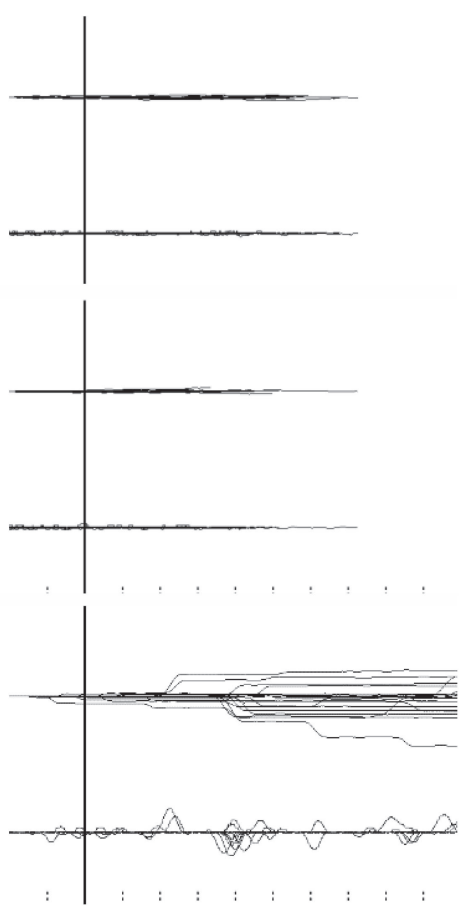

Fig. 8. Impaired inhibitory control of saccades in Parkinson's disease (PD). Recording of both saccades to cue (left) and saccades to target (right). The frequencies of saccades to cue and saccades to target are higher in PD patients than in normal subjects (top traces) and increase with disease progression. Reproduced and modified with permission from Terao et al. ${ }^{10)}$

cerebellar output, leading to the inability to adjust the saccade amplitude so that the gaze lands exactly on target; in SCD patients with pure cerebellar manifestation, the main pathology lies in Purkinje cells of the cerebellar cortex or in the deep cerebellar nuclei comprising the cerebellofugal pathway mediating the cerebellar output. ${ }^{116)-122)}$

Patients with the cerebellar type of multiple system atrophy (MSA-C) show clinical manifestations (namely, cerebellar ataxia) that are almost indistinguishable from those seen in SCD in the earliest stage. However, in contrast to SCD patients, MSA-C patients do not exhibit the prolonged deceleration phase (see Figs. 10 and 11). ${ }^{114}$ ) This may be because in MSA-C, the cerebellopetal pathway is more prominently affected, whereas the cerebellar output tract is relatively preserved in the early phase. Thus, the two disorders may be differentiated by their saccade profiles.

Saccade initiation may also be affected in cerebellar patients with pure cerebellar manifestation, although the changes in VGS and MGS latencies are relatively mild. ${ }^{84), 85), 123)}$ It is important to note that
SCD patients in advanced stages can perform MGSs with a relatively normal latency. However, saccade abnormalities vary from case to case, with no consistent pattern. There are reports that VGS latency tends to be slightly prolonged in cerebellar patients compared with normal subjects, whereas MGS latency is largely normal. ${ }^{123)}$ This makes sense considering that the cerebellum plays an important role in visually guided movements, but there are some reports to the contrary, and it is as yet unsettled whether there is a consistent pattern for cerebellar ataxia. ${ }^{82)-85)}$

c) Progressive supranuclear palsy: brainstem pattern of saccade abnormality. Progressive supranuclear palsy (PSP) is a neurological disorder associated with supranuclear gaze palsy, falls (in early stages), dysphagia, dysarthria, prominent axial rigidity, and behavioral/cognitive impairment. The main pathological feature is tau protein distribution in the brain, especially in the brainstem and the BG, leading to characteristic oculomotor manifestations due to involvement of the neural structures associated with saccades. 

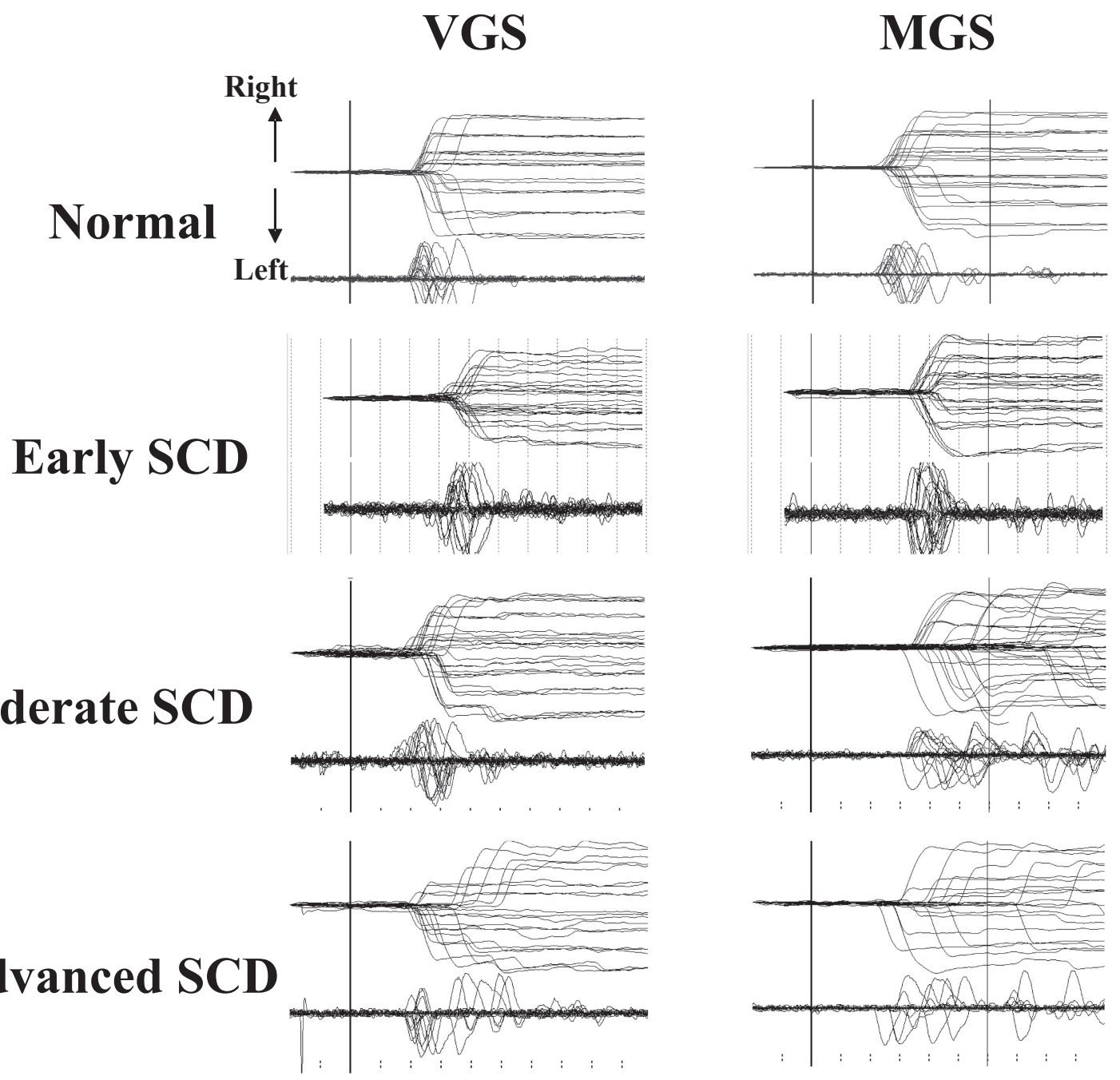

Fig. 9. Saccade records in a patient with spinocerebellar degeneration (SCD) with pure cerebellar manifestation. Saccade records of patients with SCD at various stages of progression (2nd to 4th traces) are shown in comparison with those of a normal subject (top traces). Conventions as in Fig. 7. Note the variable amplitude across trials for both visually guided saccades (VGSs) and memoryguided saccades (MGSs). In SCD patients, hypometria is evident in VGSs, whereas hypermetria is also observed in some trials for MGSs.

Figure 12A shows typical VGS and MGS records in a patient with PSP. The slope of the saccade traces, showing the velocity of the saccades, is less steep in PSP patients compared with normal subjects, indicating low saccade velocity (saccades with low velocity are termed slow saccades). The decreased velocity of saccades may also be recognized from the decreased height of the saccadic pulse in the velocity profile (lower traces in each figure). Both VGS and MGS latencies increase with disease progression, although at an early stage, VGS latency may be shorter than normal, gradually increasing with disease stage. ${ }^{6)}$
In the earliest stages of PSP, the saccade trajectory shows marked hypometria as in PD, but eventually the traces come to show a curved trajectory. ${ }^{124)}$ This may be due to the saccade having become so fragmented into sequences of a multitude of small-amplitude saccades that the gaze appears to course smoothly with a curved trajectory (Fig. 12A). ${ }^{125)}$ This feature becomes more evident with disease progression. Repeated saccade recordings at different times in the same patients also show deterioration of saccade performance over the course of 1-2 years (Fig. 12B). This may correspond to the pathology extending to various neural structures, 
A MGS 5 deg

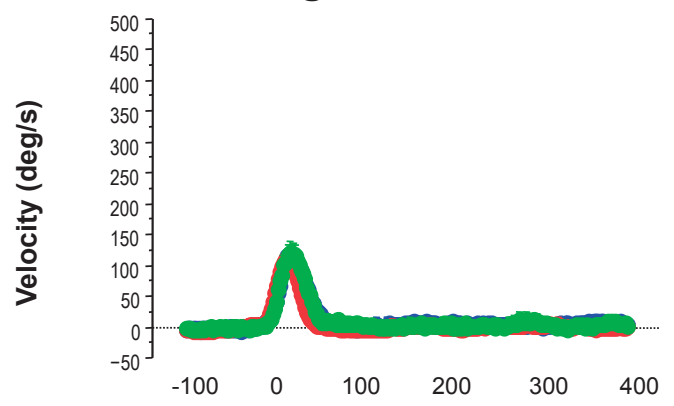

Time (ms)

\section{MGS $20 \mathrm{deg}$}

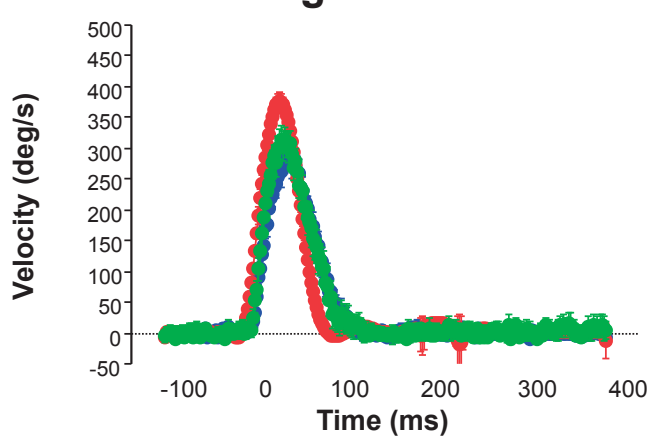

B MGS $10 \mathrm{deg}$

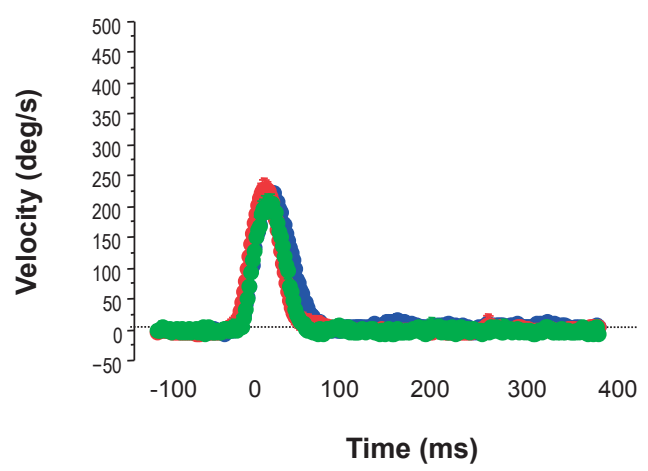

D MGS $30 \mathrm{deg}$

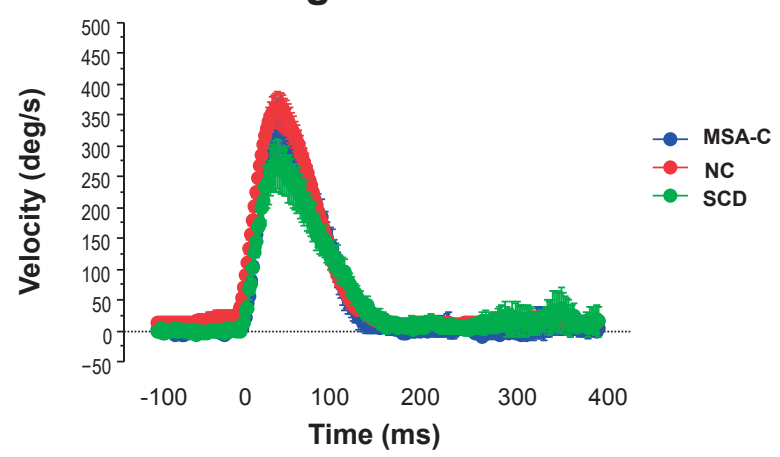

Fig. 10. Velocity profile of saccades in patients with spinocerebellar degeneration (SCD) with pure cerebellar manifestation and cerebellar type of multiple system atrophy. Subjects were asked to perform the memory-guided saccade (MGS) task, with the target presented at eccentricities of 5, 10, 20, and 30 degrees (A, B, C, and D, respectively). The velocity profiles of saccades (10-20 trials) are superimposed, aligned at the onset of the saccades $(0 \mathrm{~ms})$. The horizontal axis gives the time, and the vertical axis the velocity of saccades. Thus, SCD patients (green curves) show a lower peak and a slightly longer duration, compared with a normal control (NC, red curves) subjects and a patient with cerebellar type of multiple system atrophy (MSA-C, blue curves), especially at an eccentricity of 30 degrees. Peak velocity is also reduced in the MSA-C patient at 20-30 degrees.

including the SNr, SNc, SC, midbrain tegmentum, dentate nuclei, red nuclei, and the globus pallidus, with disease progression.

Slow saccades are saccades with reduced velocity, considering their amplitude. Physiologically, the generator of horizontal saccades is located in the PPRF, whereas vertical saccades are controlled by the riMLF in the rostral midbrain reticular formation. Supranuclear commands for volitional saccades reach the PPRF or riMLF from the frontal and parietal cortices via the SC. Slow saccades may be caused by direct disruption of neural structures generating the saccadic pulse, i.e., they may be due to intrinsic disturbance of $\mathrm{BNs}$, failure to recruit a portion of BNs, a loss of higher-level excitatory input to BNs, or even to abnormal inhibition of omnipause neurons when saccades are initiated. ${ }^{6)}$ In the case of PSP, reduced saccade velocity may also herald brainstem pathology of neural structures intimately related to saccade generation, either in the brainstem reticular formation of the midbrain tegmentum (riMLF) implicated in vertical saccades as well as the SC, but later also in PPRF for horizontal saccades. ${ }^{124)-126)}$

Slow saccades are seen not only in PSP; therefore, they are not specific to this disorder. For example, slow saccades are also encountered in Huntington's disease and spinocerebellar ataxia 2 , in which these neural structures responsible for saccade initiation are also affected. ${ }^{83)}$ However, assessing saccade velocity is useful when differentiating between PD and PSP, which is a common clinical situation. Because the peak velocity of saccades is correlated with saccade amplitude, it should be evaluated in 


\section{A Peak velocity}

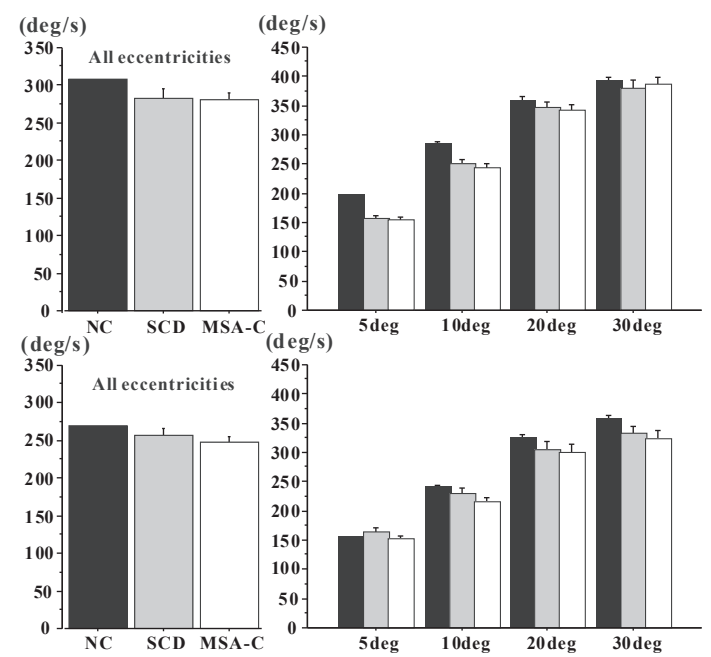

\section{B Duration}
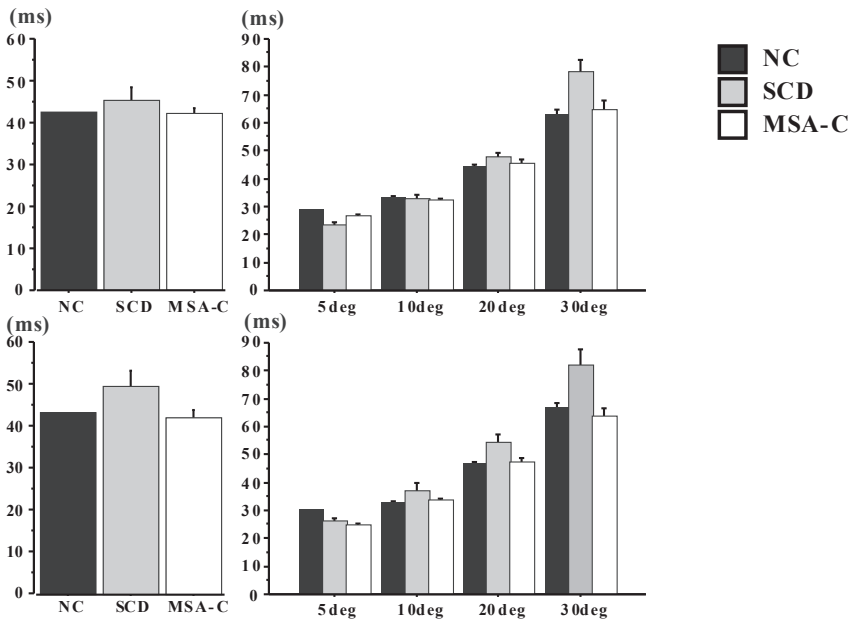

Fig. 11. Comparison of peak velocity and duration of saccades among normal subjects, spinocerebellar degeneration (SCD) patients, and cerebellar type of multiple system atrophy (MSA-C) patients. Peak velocity (A) and duration of saccades (deceleration period, B) are shown for normal control (NC) subjects, SCD patients, and MSA-C patients, for all target eccentricities (left of each figure), and separately at different target eccentricities (right of each figure, 5, 10, 20, and 30 degrees). Error bars denote standard errors. Black bars: NC, gray bars: SCD, white bars: MSA-C. Reproduced and modified with permission from Terao et al. ${ }^{124)}$

relation to saccade amplitude (main sequence relationship). ${ }^{127)}$ Although hypometria predominates in $\mathrm{PD}$ and worsens with disease progression, saccade velocity is within the normal range when taking the main sequence relationship into account. ${ }^{96)}$ In PSP, saccade velocity is reduced from an early stage in the disease course, even when considering the main sequence. It is important to note that saccade slowing can occur even in normal subjects who are tired and sleepy, so it is important to make sure that the subject is alert when recording saccades.

The inhibitory control of fixation is also affected in PSP. Patients with PSP may have difficulty holding their gaze in one position. One example are square-wave jerks (SWJs); SWJs consist of an initial saccade away from the target, followed after a short delay of approximately $200 \mathrm{msec}$ by a "return saccade" that brings the eye back to the initial location. The gaze shift away from the fixated location and the gaze shift back to the fixated location both represent a saccade. SWJs are often seen in cerebellar disorders, in which gaze fixation is frequently affected, and are thought to be generated by a mechanism similar to that of microsaccades. ${ }^{128), 129)}$ If one subpopulation of BNs is impaired, the remaining healthy $\mathrm{BNs}$ overcome the inhibition from omnipause neurons and trigger saccades.

\section{Limitations of clinical studies}

We have seen how saccade recordings in patients have been used to probe the dysfunction of the oculomotor system in patients with neurological disorders, thereby providing a way to look into the underlying pathophysiology of the patients.

This task, however, turned out to be more difficult than it first appeared. It is important to note that no reliable associations can be made between any single oculomotor parameter and a single neural system. For example, as noted above, hypometria can occur with lesions to several neural structures within the oculomotor system, and is not specific enough to any neurological condition. This is because the saccade system is subserved redundantly by various neural structures, so that dysfunction of individual neural components does not entirely abolish saccade generation, but instead leads to changes in various saccade parameters, including latency, amplitude, and velocity.

For example, Fig. 13A contrasts the latencies of reactive (VGSs and GSs) and voluntary saccades (MGSs) among patients with different neurological disorders. On average, the latencies of reactive saccade paradigms were longer in patients with cerebellar ataxia and parkinsonism, compared with 
VGS

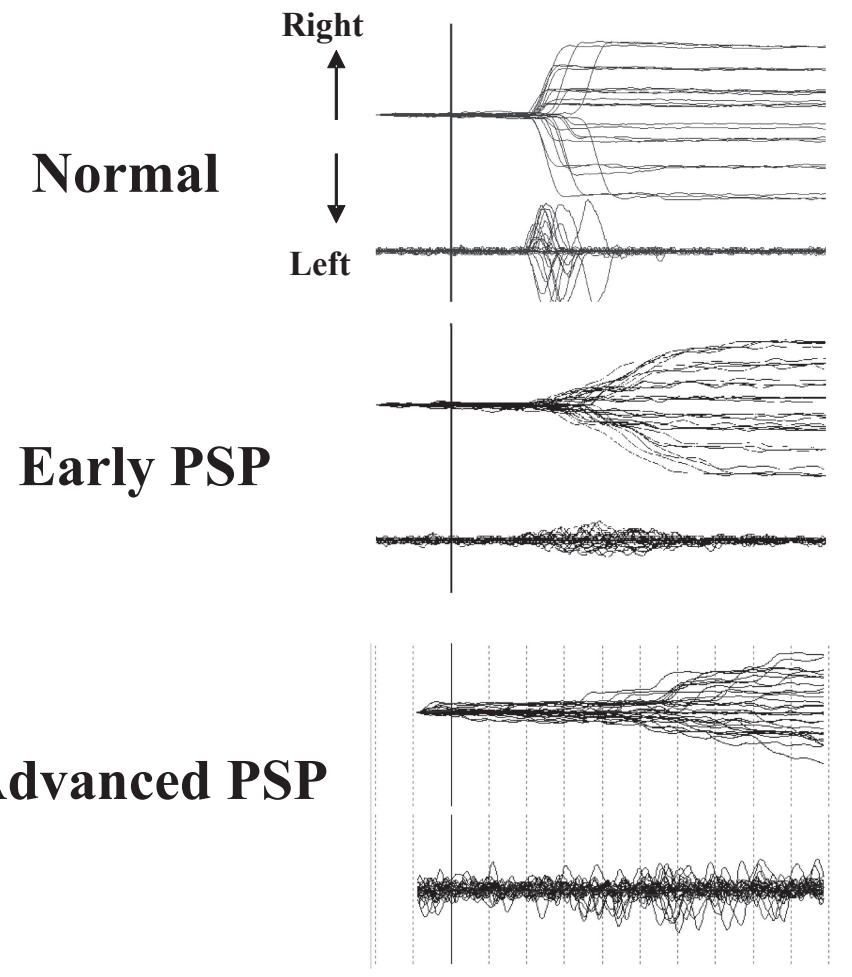

MGS
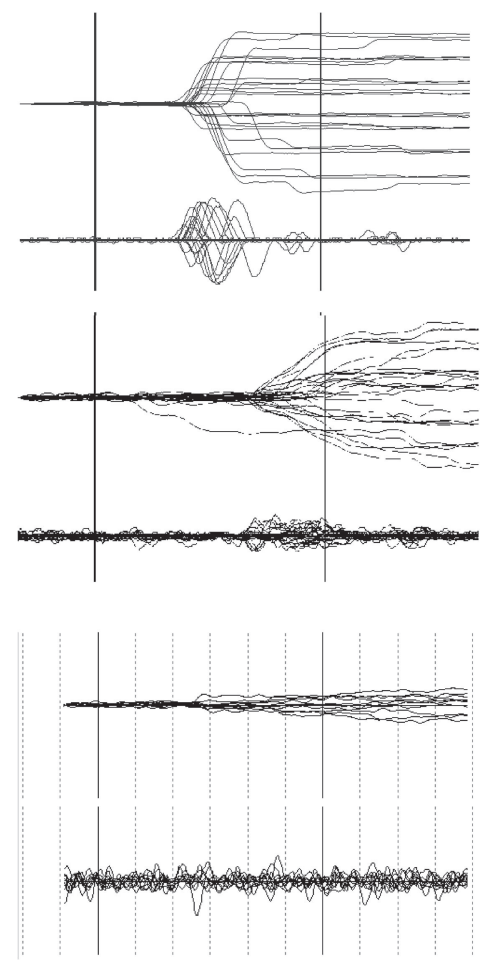

Fig. 12. Saccade records in patients with progressive supranuclear palsy. A. Traces of visually guided saccades (VGSs) (left) and memory-guided saccades (MGSs) (right) are shown for patients with progressive supranuclear palsy (PSP) at early and advanced stages (middle and bottom traces), along with those of a normal control subjects (top traces). Conventions are as in Fig. 3. Reproduced with permission from Terao et al. ${ }^{83)}$ Saccade amplitudes decrease with even lower velocity as the disease progresses, whereas the changes in latency are comparatively mild. (Continued on next page.)

normal subjects. By contrast, the latency of MGSs was normal in patients with cerebellar ataxia. However, prolongation of latency cannot be used to distinguish different neurological disorders within each disease category. Another problem is that the progression of each neurological disorder has to be taken into consideration when comparing saccade abnormalities across different neurological disorders.

Figure 13B compares saccade amplitudes across different neurological disorders. For reactive saccades (VGSs and GSs), the saccade amplitudes show hypometria, both for patients with cerebellar ataxia (yellow bars, SCD and MSA-C) and parkinsonism (green bars, PD, multiple system atrophy of the parkinsonian type [MSA-P], and PSP). By contrast, for voluntary saccade tasks such as the MGS task, saccade amplitude is normal on average in patients with cerebellar ataxia, but hypometric in patients with parkinsonism. Therefore, hypometria appears to be more "consistently" observed in parkinsonism than in cerebellar ataxia. However, even hypometria does not help to differentiate between different disorders within each category of parkinsonism.

The success rate of MGSs is lower in patients with parkinsonism than in patients with cerebellar ataxia, who show largely normal success rates on average, but again, disorders within each category cannot be distinguished based on MGS success rates (Fig. 14A). The ability for inhibitory control of saccades as reflected in the frequency of saccades to cue and saccades to target is similarly impaired in patients with parkinsonism and cerebellar ataxia, as well as in those with focal lesions (Figs. 14B and C), and cannot reliably be used to differentiate these different neurological disorders, either.

Therefore, the entire pattern of saccade abnormalities has to be considered, rather than focusing on any single saccade parameter (Table 1 ). In clinical 
B

Case 1

\section{First visit}

VGS

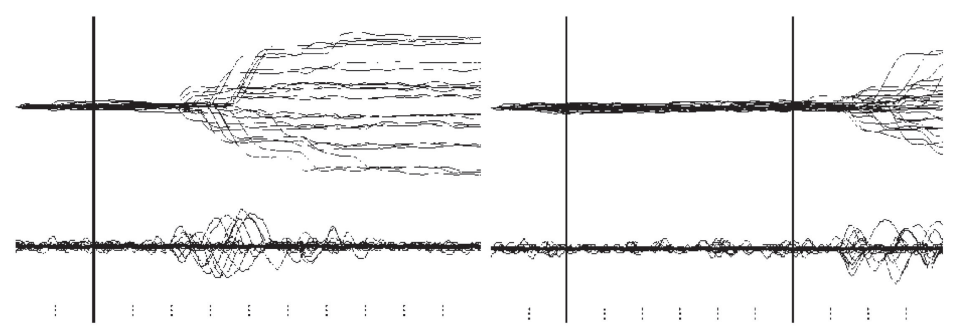

One year later

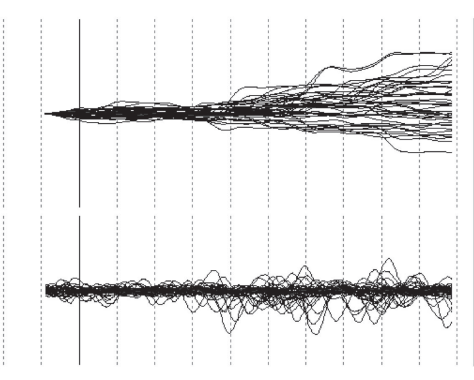

MGS

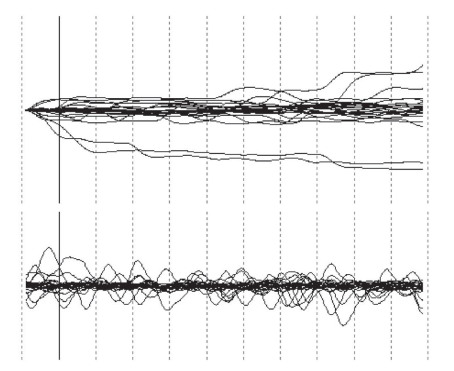

Case 2

\section{First visit}

Two years later

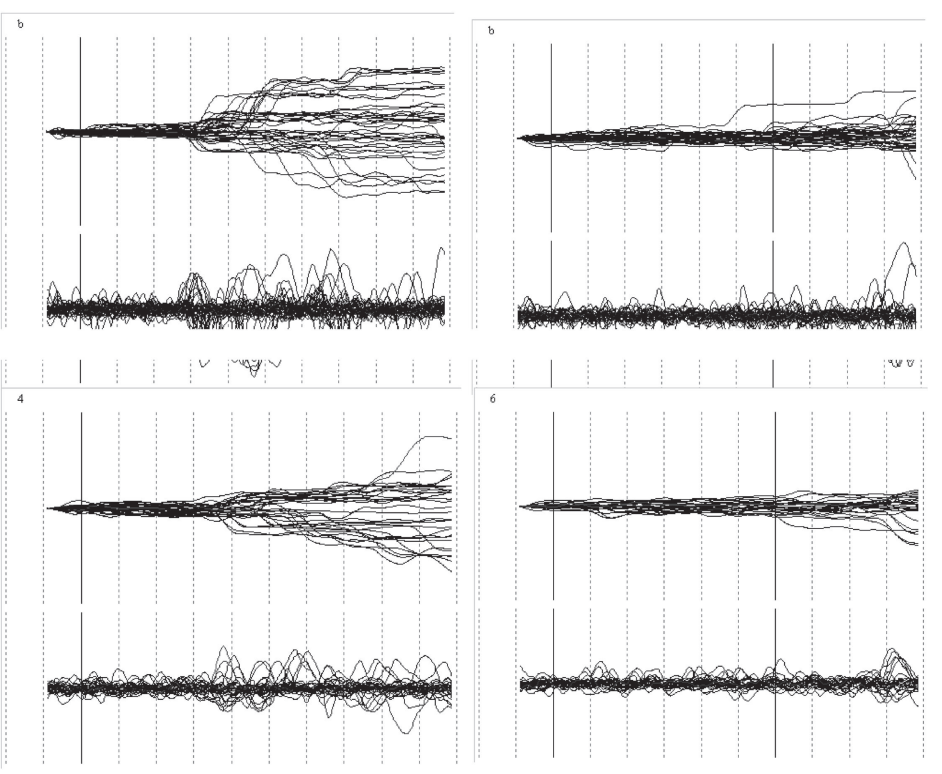

Fig. 12. B. Repeated saccade recording at an interval of 1-2 years in two patients with PSP. Note the remarkable reduction in saccade amplitude from the first to the second recording. Reproduced with permission from Terao et al. ${ }^{124)}$

practice, if the saccade abnormality in a patient shows a pattern similar to a pattern already reported in a neurological disorder, we may infer that the underlying pathophysiology (and the diagnosis) would also be similar. For example, overall hypometria in any saccade task, plus the preferential involvement of voluntary saccades relative to reactive saccades, would suggest parkinsonism. However, some patients may not show such specifics of already known saccade abnormality patterns. How can we infer the pathophysiology from such saccade records? In practice, as a first step, we may have to determine all the major patterns of saccade abnormalities associated with neurological disorders. These major patterns may be called "principal" components of the saccade abnormality, with a full understanding how the underlying pathophysiology leads to the abnormalities. For example, we have not yet found a 

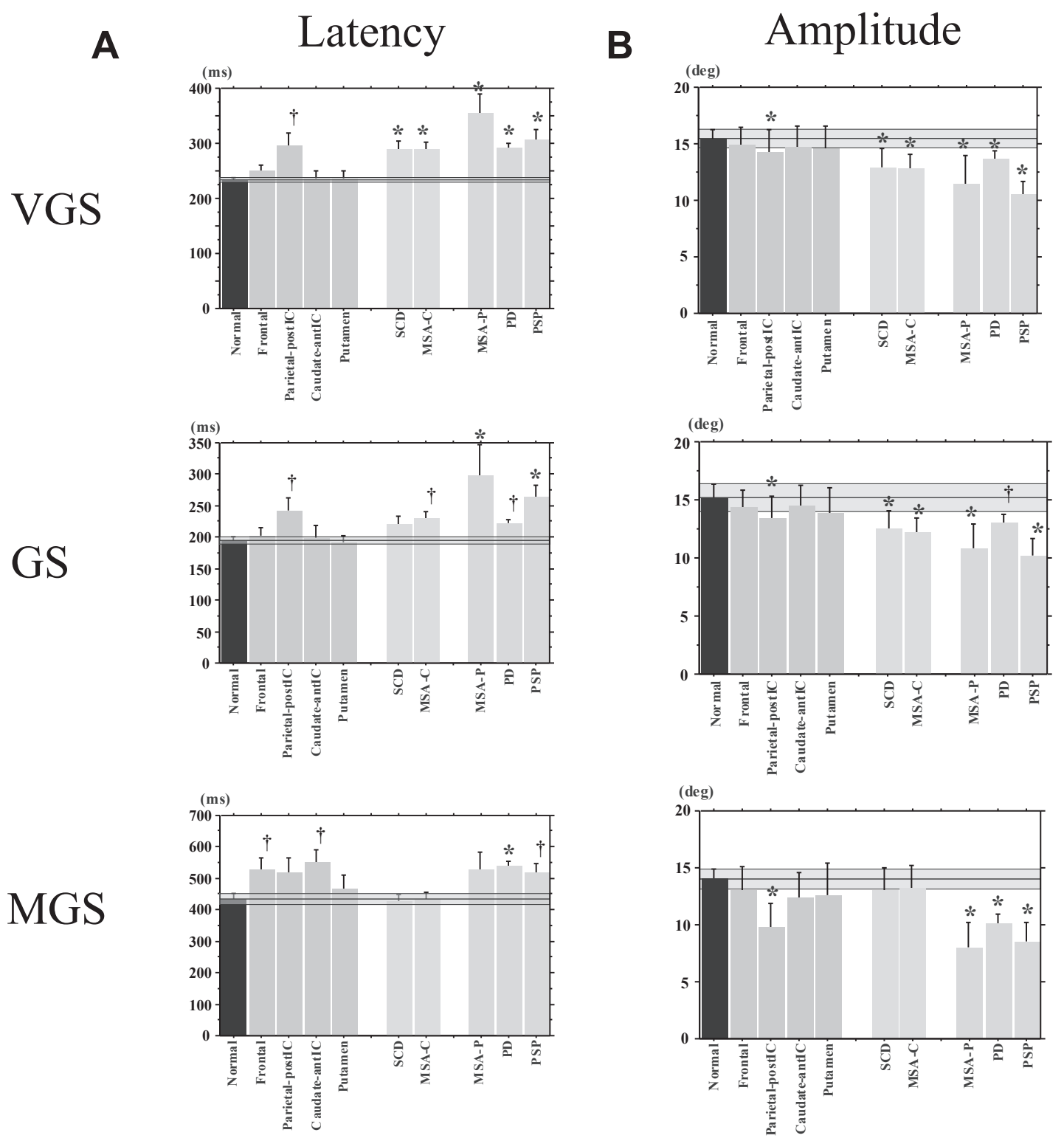

Fig. 13. Latency and amplitude of reflexive saccades in various neurological disorders. The latency $(\mathbf{A})$ and amplitude $(\mathbf{B})$ of saccades in the visually guided saccade (VGS; upper figures), gap saccade (GS; middle figures), and memory-guided saccade (MGS) tasks (bottom figures) are shown for normal controls (left-most bar) and various neurological disorders. Frontal: frontal lesion, ParietalpostIC: lesion in the parietal cortex and/or posterior limb of the internal capsule, Caudate-antIC: lesion in the caudate nucleus and/or anterior limb of the internal capsule, Putamen: putaminal lesion, SCD: spinocerebellar degeneration (pure cerebellar manifestation), MSA-C: multiple system atrophy (cerebellar type), MSA-P: multiple system atrophy (parkinsonian type), PD: Parkinson's disease, PSP: progressive supranuclear palsy. The transparent blue areas denote the $95 \%$ confidence range in the age-matched control subjects. The daggers and asterisks indicate a significant difference from normal controls before and after correction, respectively, for multiple comparisons. Error bars give the standard errors (also in the following figures).

specific pattern associated with cerebellar ataxia, presumably because of miscellaneous patho(physio)logies in the studied cases. However, even with the knowledge of miscellaneous patterns, there may be cases in which the pattern of the saccade abnormality does not entirely match any known pattern. In these cases, we may try to determine whether the saccade pattern can be explained as a "combination" of basic patterns already known. Although extensive studies have been conducted, we do not yet know whether this can be achieved, and this issue requires further investigation. 


\section{A}

MGS success rate

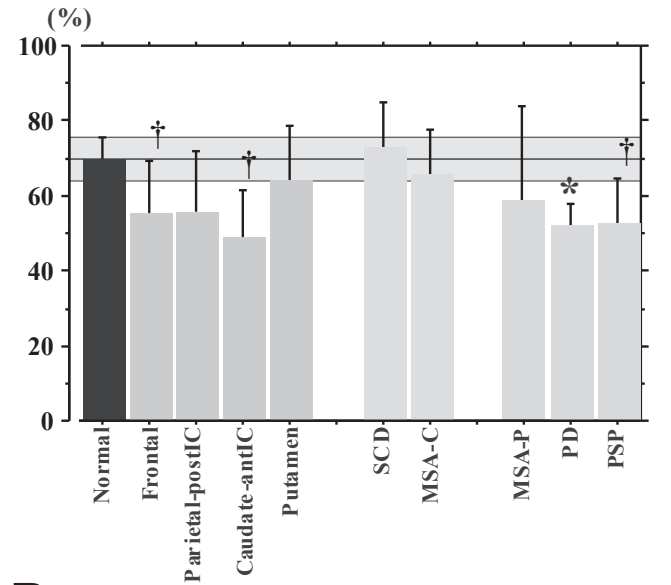

B Saccades to cue

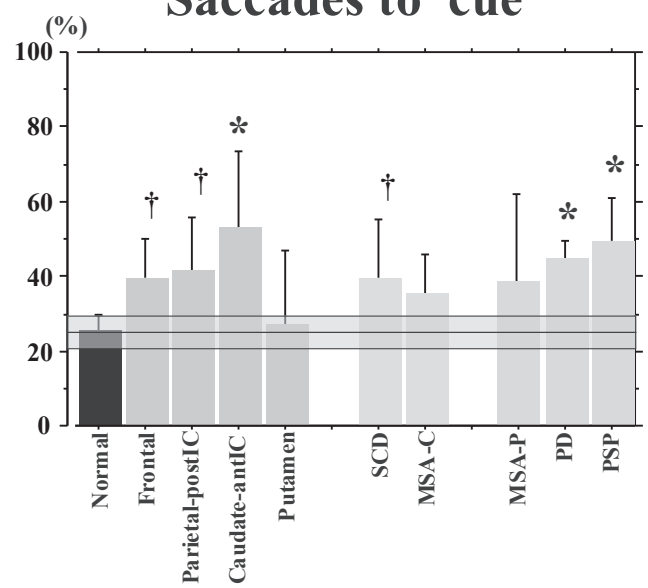

\section{Saccades to target}

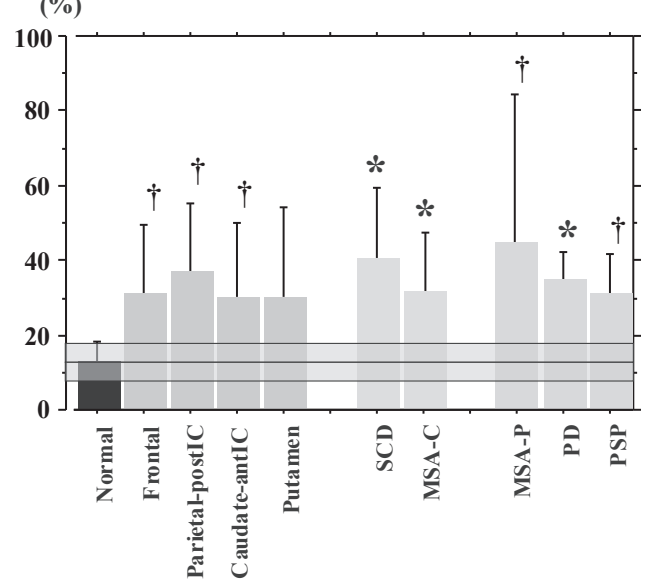

Fig. 14. Memory-guided saccade (MGS) success rate, frequencies of saccades to cue, saccades to target, and amplitude of MGS in various neurological disorders. MGS success rate (A), frequencies of saccades to cue (B), as well as saccades to target $(\mathbf{C})$ are shown for normal controls and various neurological disorders, following conventions of the previous figure.

\section{Insights gained from interfering with the saccade control system}

Most of the clinical studies in neurological patients have investigated the "stationary condition" of saccade abnormalities. However, interfering with the saccade system and looking at the changes in saccade performance may tell us more about the functioning of the oculomotor system at the systems level.

Using deep brain stimulation (DBS), it is possible to interfere with the processing of saccade performance subcortically. ${ }^{130)-137)}$ For example, Yugeta et al. ${ }^{130)}$ investigated the performances of reactive (VGSs and GSs) and voluntary saccade paradigms (MGSs and ASs) in PD patients treated with DBS of the subthalamic nucleus (STN DBS).
The authors found that, by switching DBS on and off, the hypometric saccades typically noted in PD patients were improved when DBS was turned on, relative to when it was turned off, for all the saccade paradigms studied, i.e., both reactive (VGSs and GSs) and voluntary (MGSs and ASs) (Fig. 15). Saccade latencies in reactive saccade tasks were also shortened by DBS, but not in voluntary saccades. By contrast, the frequency of saccades to cue, i.e., unwanted reactive saccades made to the presentation of a cue that signals the future target location in the MGS task, decreased after DBS. Thus, both the initiation and inhibitory control of saccades were affected by DBS. The authors concluded that DBS possibly works by acting on the STN-SNr-SC pathway relatively directly, changing the excitability of the SC; in other words, STN DBS affects the 
neural pathway common to both reflexive and volitional saccades, and may set the functional level of the SC at a level appropriate for both saccade initiation and inhibition. ${ }^{89)}$

\section{Saccadic eye movements in daily life; can they be used for clinical examination?}

So far, we have reviewed the typical patterns of saccade abnormalities in major neurological disorders and how they relate to the underlying pathophysiology. However, to ensure that the saccade abnormalities are not just a specific finding obtained under experimental conditions but have some behavioral correlates in daily life, we need to investigate what difficulties a specific saccade abnormality causes for patients in their daily activities. This would require studies using eye tracking methodologies to be performed in a more natural setting, rather than in a laboratory setting. ${ }^{12)}$

As mentioned at the beginning of this review, we move our eyes using saccades alternated with fixation as we collect visual information from the outer world. Using eye tracking, Yarbus et al. investigated how people look at another person's face. ${ }^{1)}$ They do not scan all around the face, but focus on relatively restricted regions, such as the eyes, the mouth, and the circumference of the face. How do patients with neurological disorders scan the world with their eyes, and how does this differ from normal subjects? Eye tracking technology has also been put to clinical use in a wide range of disorders. An early application in neurological patients is hemispatial neglect. Hemispatial neglect is characterized by reduced awareness of stimuli on one side of space. Eye tracking has shown that patients with left hemispatial neglect fail to explore items to their left, and are often unaware of objects or people on that side, e.g., when they are shown a display with the letter $\mathrm{T}$ embedded among rotated $\mathrm{Ls}$ and are instructed to find the Ts. ${ }^{138)-141)}$ Notably, the presence or absence of homonymous hemianopia or hemispatial neglect did not affect spatial patterns of gaze restricted to the left hemispace.

Eye tracking has also been applied to visual perception and language comprehension. When normal subjects read an English text, they do not read the letters one by one, but the gaze leaps from one word to the next. They look at each word only once or at most twice, with the gaze landing typically at a position shifted slightly to the right of the word center. Longer words often receive more than one fixation, but shorter words and words that are 

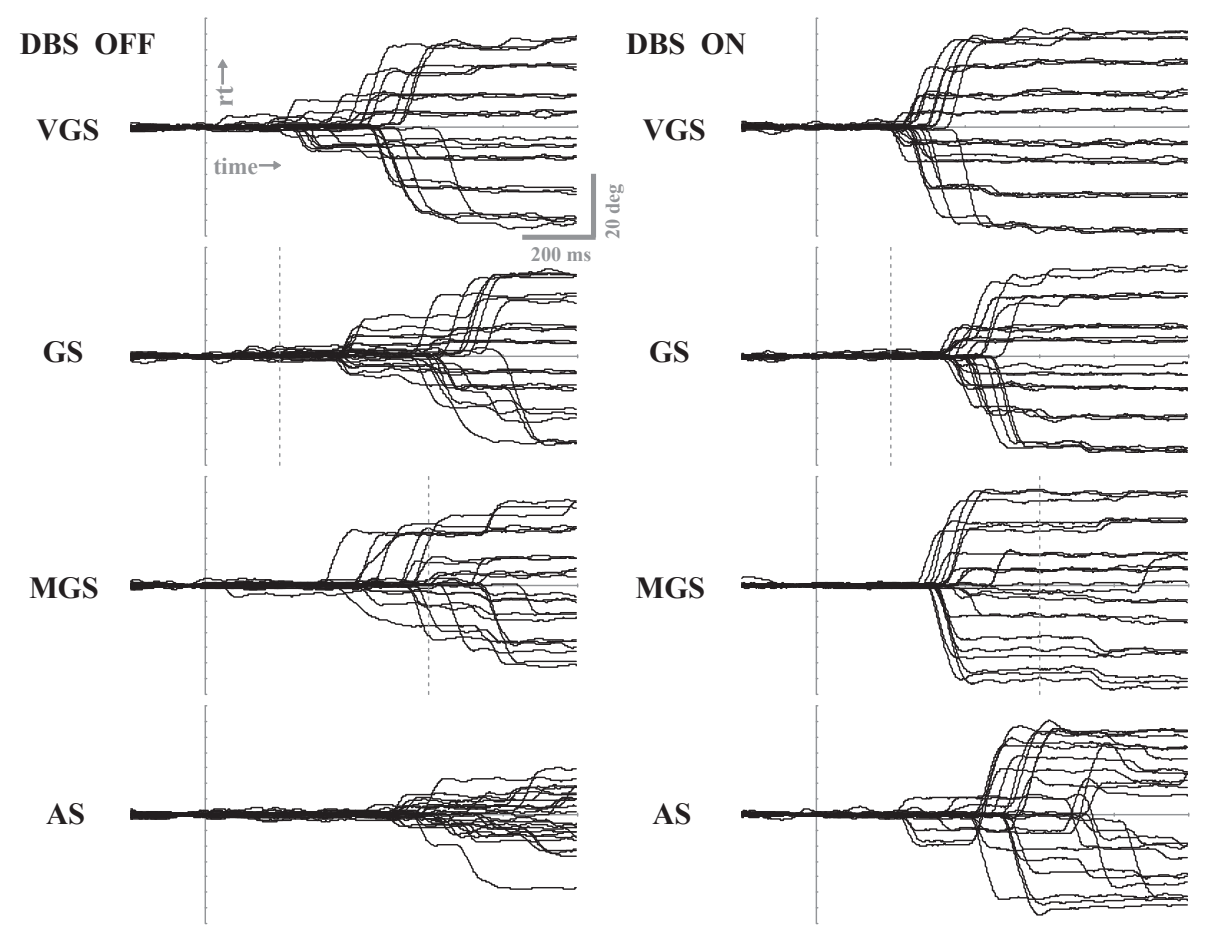

Fig. 15. Saccade performances of oculomotor tasks when deep brain stimulation was turned off (left) and on (right) in a PD patient. Traces for 20-30 trials are superimposed for four oculomotor tasks: visually guided saccade (VGS), gap saccade (GS), memory-guided saccade (MGS), and antisaccade (AS). Vertical bars indicate the time signaling the start of saccades. Note the increased amplitude for all tasks when deep brain stimulation (DBS) is switched on (right) as compared to when it is off (left). Reproduced and modified with permission from Yugeta et al. $\left.{ }^{130}\right)$

grammatically predictable are often skipped altogether. $^{142)}$ By contrast, eye tracking shows that patients with pure alexia, if not letter-by-letter, proceed slowly by looking at $2-3$ letters at a time, a pattern that is not observed in normal subjects. ${ }^{143)}$

Foulsham et al. ${ }^{144)}$ conducted eye tracking in a patient with visual agnosia while she searched for objects within photographs of natural scenes, and her eye movements were compared with those of students and age-matched controls. For example, subjects were shown a scene of a room on the computer screen and instructed to find a target object (e.g., an apple on the desk). The patient with visual agnosia made recursive and redundant gaze movements that were drawn to more salient parts of the scene, and resorted to bottom-up searching relying on a perceptual cue like red color, but was not capable of top-down searching for the target object.

Mosiman et al. ${ }^{145)}$ recorded gaze patterns while patients with Alzheimer's disease (AD) and normal subjects viewed a line drawing of a clock to read out the time. In normal subjects, fixation was most frequently distributed around the points of the clock hands. In contrast, although patients with $\mathrm{AD}$ also looked at the clock hands; their gaze was more focused around the center of the clock, which normal subjects almost never looked at. Furthermore, AD patients first looked at the points of the clock hands later than normal subjects. In contrast, the latency and amplitude of VGSs were similar to those in normal subjects. This implies that AD patients had normal abilities to initiate reflexive saccades to a suddenly appearing target, but were impaired in active exploration of the scene.

Recently, eye tracking has been applied to reallife social interactions. In a head-unrestrained setup, Spezio et al. ${ }^{146)}$ recorded gaze movements while subjects faced other people during everyday conversations. They studied eye contact when patients with bilateral amygdalar damage, who were impaired in judging the emotions shown in photographs of faces, an impairment caused by a failure to fixate on informative facial features, made conversation with other people. ${ }^{147)}$ They found that the gaze was more frequently directed at the mouth, but not at the eyes, as is usual for normal subjects. The same pattern of gaze movement has also been described repeatedly in patients with autism. ${ }^{148)}$ The diminished eye-looking 
in amygdalar damage and autism was thought to reflect passive insensitivity to the social signals in others' eyes.

Here we provide an example of our eye tracking study applied to neurological disorders. As discussed above, patients with parkinsonism show hypometric saccades in almost all saccade paradigms performed in an experimental setting. What problem does this abnormality cause in PD patients in daily life, such as when they move their eyes to scan the visual world in front of them? Matsumoto et al. ${ }^{149)}$ asked patients with PD to view line drawings of various complexity presented for $10 \mathrm{sec}$ (memorization period) on a computer screen, and to reproduce them on a piece of paper after the drawings were hidden. When the gaze movement was recorded during the memorization period using a video-based eye tracking system, the area of the images that the patients scanned was significantly smaller than that scanned by normal subjects performing the same task, as was the saccade amplitude during the scanning of the images, and the frequency of saccades per unit of time. Thus, gaze movements made in daily life likely have characteristics similar to not only those demonstrated in experimental oculomotor tasks, but also to the motor symptoms of the patients, i.e., akinesia and bradykinesia. Importantly, the decreased saccade amplitude contributed more to the reduction of the scanned area in PD patients than the frequency of saccades per unit of time or the mean fixation period.

Some patients have difficulty performing conventional oculomotor tasks in an experimental setting, even simple ones that normal subjects perform without difficulty. Another approach would be to observe a large number of eye movements made during a task that almost every subject could perform without any specific effort, e.g., a free viewing task, and to analyze all the gaze movements made during this task for diagnosis. Based on the assumption that natural attention and eye movement behavior contain a biometric signature of an individual and of her/his state of brain function or dysfunction, Tseng et al. ${ }^{150)}$ extracted quantitative features from the gaze data using automated machine learning in a workflow inspired by microarray analysis. These quantitative features included oculomotor-based core features and saliency-based core features associated with attention from the saccade gaze traces. Based on this automated procedure, the authors were able to differentiate patients with attention deficit hyperactivity disorder (ADHD) and fetal alcohol spectrum disorder (FASD) from control subjects, based on a large number of saccades made during this free viewing task. The authors were even able to differentiate between ADHD and FASD patients who have overlapping behavioral phenotypes. Despite the efficiency of this procedure and its potential as a promising method for extracting gaze signatures as potential biomarkers of particular neurological disorders, the physiological significance and behavioral correlates of the signatures require further investigation.

\section{Acknowledgements}

Dr. Terao received support from a Research Project Grant-in-aid for Scientific Research from the Ministry of Education, Culture, Sports, Science and Technology of Japan (16K09709, 16H01497).

\section{References}

1) Yarbus, A.L. (1967) Eye Movements and Vision (B. Haigh, Trans.). Plenum Press, New York.

2) Rayner, K. (2009) Eye movements and landing positions in reading: a retrospective. Perception 38, 895-899.

3) Gilchrist, I.D., Brown, V. and Findlay, J.M. (1997) Saccades without eye movements. Nature 390 , 130-131.

4) Willmore, B.D., Mazer, J.A. and Gallant, J.L. (2011) Sparse coding in striate and extrastriate visual cortex. J. Neurophysiol. 105, 2907-2919.

5) Hayhoe, M. and Ballard, D. (2005) Eye movements in natural behavior. Trends Cogn. Sci. 9, 188-194.

6) Leigh, R.J. and Kennard, C. (2004) Using saccades as a research tool in the clinical neurosciences. Brain 127, 460-477.

7) Ramat, S., Leigh, R.J., Zee, D.S. and Optican, L.M. (2007) What clinical disorders tell us about the neural control of saccadic eye movements. Brain 130, 10-35.

8) Johnston, K. and Everling, S. (2008) Neurophysiology and neuroanatomy of reflexive and voluntary saccades in non-human primates. Brain Cogn. 68, 271-283.

9) Terao, Y., Fukuda, H., Tokushige, S., InomataTerada, S. and Ugawa, Y. (2017) How saccade intrusions affect subsequent motor and oculomotor actions. Front. Neurosci. 10, e608.

10) Terao, Y., Fukuda, H., Yugeta, A., Hikosaka, O., Nomura, Y., Segawa, M., Hanajima, T., Tsuji, S. and Ugawa, Y. (2011) Initiation and inhibitory control of saccades with the progression of Parkinson's disease - changes in three major drives converging on the superior colliculus. Neuropsychologia 49, 1794-1806.

11) Braun, D., Weber, H., Mergner, T. and SchulteMönting, J. (1992) Saccadic reaction times in patients with frontal and parietal lesions. Brain 115, 1359-1386.

12) Popa, L., Selejan, O., Scott, A., Mureşanu, D.F., 
Balea, M. and Rafila, A. (2015) Reading beyond the glance: eye tracking in neurosciences. Neurol. Sci. 36, 683-688.

13) Houben, M.M., Goumans, J. and van der Steen, J. (2006) Recording three-dimensional eye movements: scleral search coils versus video oculography. Invest. Ophthalmol. Vis. Sci. 47, 179-187.

14) Hikosaka, O. and Wurtz, R.H. (1989) The basal ganglia. Rev. Oculomot. Res. 3, 257-281.

15) Müri, R.M. and Nyffeler, T. (2008) Neurophysiology and neuroanatomy of reflexive and volitional saccades as revealed by lesion studies with neurological patients and transcranial magnetic stimulation (TMS). Brain Cogn. 68, 284-292.

16) McDowell, J.E., Dyckman, K.A., Austin, B.P and Clementz, B.A. (2008) Neurophysiology and neuroanatomy of reflexive and volitional saccades: evidence from studies of humans. Brain Cogn. 68, 255-270.

17) Munoz, D.P. (2002) Commentary; Saccadic eye movements: overview of neural activity. Prog. Brain Res. 140, 89-96.

18) Gaymard, B., Pierrot-Deseilligny, C. and Rivaud, S. (1990) Impairment of sequences of memoryguided saccades after supplementary motor area lesions. Ann. Neurol. 28, 622-626.

19) Gaymard, B., Rivaud, S. and Pierrot-Deseilligny, C. (1993) Role of the left and right supplementary motor areas in memory-guided saccade sequences. Ann. Neurol. 34, 404-406.

20) Hallett, P.E. (1978) Primary and secondary saccades to goals defined by instructions. Vision Res. 18, 1279-1296.

21) Curtis, C.E. and D'Esposito, M. (2003) Success and failure suppressing reflexive behavior. J. Cogn. Neurosci. 15, 409-418.

22) Munoz, D.P., Armstrong, I.T., Hampton, K.A. and Moore, K.D. (2003) Altered control of visual fixation and saccadic eye movements in attentiondeficit hyperactivity disorder. J. Neurophysiol. 90, 503-514.

23) Munoz, D.P. and Everling, S. (2004) Look away, the anti-saccade task and the voluntary control of eye movement. Nat. Rev. Neurosci. 5, 218-228.

24) Brown, M.R., Goltz, H.C., Vilis, T., Ford, K.A. and Everling, S. (2006) Inhibition and generation of saccades: rapid event-related fMRI of prosaccades, antisaccades, and nogo trials. Neuroimage $\mathbf{3 3}$ 644-659.

25) Jamadar, S.D., Fielding, J. and Egan, G.F. (2013) Quantitative meta-analysis of fMRI and PET studies reveals consistent activation in frontostriatal-parietal regions and cerebellum during antisaccades and prosaccades. Front. Psychol. 4 749 .

26) Herweg, N.A., Weber, B., Kasparbauer, A., Meyhöfer, I., Steffens, M., Smyrnis, N. and Ettinger, U. (2014) Functional magnetic resonance imaging of sensorimotor transformations in saccades and antisaccades. Neuroimage 102, 848860.

27) Bruce, C.J. and Goldberg, M.E. (1985) Primate frontal eye fields. I. Single neurons discharging before saccades. J. Neurophysiol. 53, 603-635.

28) Goldberg, M.E. and Bruce, C.J. (1990) Primate frontal eye fields. III. Maintenance of a spatially accurate saccade signal. J. Neurophysiol. 64, 489508.

29) Mountcastle, V.B., Lynch, J.C., Georgopoulos, A., Sakata, H. and Acuna, C. (1975) Posterior parietal association cortex of the monkey: command functions for operations within extrapersonal space. J. Neurophysiol. 38, 871-908.

30) Lynch, J.C., Mountcastle, V.B., Talbot, W.H. and Yin, T.C. (1977) Parietal lobe mechanisms for directed visual attention. J. Neurophysiol. 40, 362-389.

31) Paré, M. and Wurtz, R.H. (1997) Monkey posterior parietal cortex neurons antidromically activated from superior colliculus. J. Neurophysiol. 78, 3493-3497.

32) Kurylo, D.D. and Skavenski, A.A. (1991) Eye movements elicited by electrical stimulation of area PG in the monkey. J. Neurophysiol. 65, $1243-1253$

33) Shibutani, H., Sakata, H. and Hyvärinen, J. (1984) Saccade and blinking evoked by microstimulation of the posterior parietal association cortex of the monkey. Exp. Brain Res. 55, 1-8.

34) Thier, P. and Andersen, R.A. (1996) Electrical microstimulation suggests two different forms of representation of head-centered space in the intraparietal sulcus of rhesus monkeys. Proc. Natl. Acad. Sci. U.S.A. 93, 4962-4967.

35) Stanton, G.B., Deng, S.Y., Goldberg, M.E. and McMullen, N.T. (1989) Cytoarchitectural characteristic of the frontal eye fields in macaque monkeys. J. Comp. Neurol. 282, 415-427.

36) Shook, B.L., Schlag-Rey, M. and Schlag, J. (1990) Primate supplementary eye field: I. Comparative aspects of mesencephalic and pontine connections. J. Comp. Neurol. 301, 618-642.

37) Grosbras, M.H., Lobel, E., Van de Moortele, P.F., LeBihan, D. and Berthoz, A. (1999) An anatomical landmark for the supplementary eye fields in human revealed with functional magnetic resonance imaging. Cereb. Cortex 9, 705-711.

38) Gaymard, B., Lynch, J., Ploner, C.J., Condy, C. and Rivaud-Péchoux, S. (2003) The parieto-collicular pathway: anatomical location and contribution to saccade generation. Eur. J. Neurosci. 17, 15181526.

39) Sweeney, J.A., Mintun, M.A., Kwee, S., Wiseman, M.B., Brown, D.L., Rosenberg, D.R. and Carl, J.R. (1996) Positron emission tomography study of voluntary saccadic eye movements and spatial working memory. J. Neurophysiol. 75, 454-458.

40) O'Driscoll, G.A., Alpert, N.M., Matthysse, S.W., Levy, D.L., Rauch, S.L. and Holzman, P.S. (1995) Functional neuroanatomy of antisaccade eye movements investigated with positron emission tomography. Proc. Natl. Acad. Sci. U.S.A. 92, 925-929.

41) Parton, A., Nachev, P., Hodgson, T.L., Mort, D., 
Thomas, D., Ordidge, R., Morgan, P.S., Jackson, S., Rees, G. and Husain, M. (2007) Role of the human supplementary eye field in the control of saccadic eye movements. Neuropsychologia 45, 997-1008.

42) Andersen, R.A., Snyder, L.H., Bradley, D.C. and Xing, J. (1997) Multimodal representation of space in the posterior parietal cortex and its use in planning movements. Annu. Rev. Neurosci. 20, 303-330.

43) Robinson, F.R. and Fuchs, A.F. (2001) The role of the cerebellum in voluntary eye movements. Annu. Rev. Neurosci. 24, 981-1004.

44) Brown, J.W., Bullock, D. and Grossberg, S. (2004) How laminar frontal cortex and basal ganglia circuits interact to control planned and reactive saccades. Neural Netw. 17, 471-510.

45) Doricchi, F., Perani, D., Incoccia, C., Grassi, F., Cappa, S.F., Bettinardi, V., Galati, G., Pizzamiglio, L. and Fazio, F. (1997) Neural control of fast-regular saccades and antisaccades, an investigation using positron emission tomography. Exp. Brain Res. 116, 50-62.

46) O'Sullivan, E.P., Jenkins, I.H., Henderson, L., Kennard, C. and Brooks, D.J. (1995) The functional anatomy of remembered saccades: a PET study. Neuroreport 6, 2141-2144.

47) Brown, M.R., DeSouza, J.F., Goltz, H.C., Ford, K., Menon, R.S., Goodale, M.A. and Everling, S. (2004) Comparison of memory- and visually guided saccades using event-related fMRI. J. Neurophysiol. 91, 873-889.

48) Gaymard, B., Ploner, C.J., Rivaud, S., Vermersch, A.I. and Pierrot-Deseilligny, C. (1998) Cortical control of saccades. Exp. Brain Res. 123, 159163

49) Heide, W. and Kömpf, D. (1998) Combined deficits of saccades and visuo-spatial orientation after cortical lesions. Exp. Brain Res. 123, 164-171.

50) Leigh, R.J. and Zee, D.S. (2006) The Neurology of Eye Movements. 4th ed. Oxford University Press, Oxford.

51) Terao, Y., Fukuda, H., Tokushuge, S., Nomura, Y., Hanajima, R. and Ugawa, Y. (2016) Saccade abnormalities associated with focal cerebral lesions - How cortical and basal ganglia commands shape saccades in humans - Clin. Neurophysiol. 127, 2953-2967.

52) Gaymard, B., Lynch, J., Ploner, C.J., Condy, C. and Rivaud-Péchoux, S. (2003) The parieto-collicular pathway: anatomical location and contribution to saccade generation. Eur. J. Neurosci. 17, 15181526.

53) Pierrot-Deseilligny, C., Rivaud, S. and Gaymard, B. (1991) Cortical control of reflexive visually guided saccades in man. Brain 114, 1473-1485.

54) Pierrot-Deseilligny, C., Rivaud, S., Penet, C. and Rigolet, M.H. (1987) Latencies of visually guided saccades in unilateral hemispheric cerebral lesions. Ann. Neurol. 21, 138-148.

55) Pierrot-Deseilligny, C., Rivaud, S., Gaymard, B. and Agid, Y. (1991) Cortical control of memory guided saccades in man. Exp. Brain Res. 83, 607617.

56) Gaymard, B., François, C., Ploner, C.J., Condy, C. and Rivaud-Péchoux, S. (2003) A direct prefrontotectal tract against distractibility in the human brain. Ann. Neurol. 53, 542-545.

57) Condy, C., Rivaud-Péchoux, S., Ostendorf, F., Ploner, C.J. and Gaymard, B. (2004) Neural substrate of antisaccades: role of subcortical structures. Neurology 63, 1571-1578.

58) Hikosaka, O., Takikawa, Y. and Kawagoe, R. (2000) Role of the basal ganglia in the control of purposive saccadic eye movements. Physiol. Rev. 80, 953-978.

59) Stein, J.F. and Glickstein, M. (1992) Role of the cerebellum in visual guidance of movement. Physiol. Rev. 72, 967-1017.

60) Thier, P. and Möck, M. (2006) The oculomotor role of the pontine nuclei and the nucleus reticularis tegmenti pontis. Prog. Brain Res. 151, 293-320.

61) Noda, H., Sugita, S. and Ikeda, Y. (1990) Afferent and efferent connections of the oculomotor region of the fastigial nucleus in the macaque monkey. J. Comp. Neurol. 302, 330-348.

62) Ohtsuka, K. and Noda, H. (1990) Direction-selective saccadic-burst neurons in the fastigial oculomotor region of the macaque. Exp. Brain Res. 81, 659662.

63) Fuchs, A.F., Robinson, F.R. and Straube, A. (1993) Role of the caudal fastigial nucleus in saccade generation. I. Neuronal discharge pattern. J. Neurophysiol. 70, 1723-1740.

64) Robinson, F.R., Straube, A. and Fuchs, A.F. (1993) Role of the caudal fastigial nucleus in saccade generation. II. Effects of muscimol inactivation. J. Neurophysiol. 70, 1741-1758.

65) Scudder, C.A., Kaneko, C.S. and Fuchs, A.F. (2002) The brainstem burst generator for saccadic eye movements: modern synthesis. Exp. Brain Res. 142, 439-462.

66) Buzunov, E., Mueller, A., Straube, A. and Robinson, F.R. (2013) When during horizontal saccades in monkey does cerebellar output affect movement? Brain Res. 1503, 33-42.

67) Dean, P. (1995) Modelling the role of the cerebellar fastigial nuclei in producing accurate saccades: the importance of burst timing. Neuroscience 68, 1059-1077.

68) Hodgson, T., Chamberlain, M., Parris, B., James, M., Gutowski, N., Husain, M. and Kennard, C. (2007) The role of the ventrolateral frontal cortex in inhibitory oculomotor control. Brain 130, 1525-1537.

69) Guitton, D., Buchtel, H.A. and Douglas, R.M. (1985) Frontal lobe lesions in man cause difficulties in suppressing reflexive glances and in generating goal-directed saccades. Exp. Brain Res. 58, 455-472.

70) Tinsley, C.J. and Everling, S. (2002) Contribution of the primate prefrontal cortex to the gap effect. Prog. Brain Res. 140, 61-72.

71) Mountcastle, V.B., Andersen, R.A. and Motter, 
B.C. (1981) The influence of attentive fixation upon the excitability of the light-sensitive neurons of the posterior parietal cortex. J. Neurosci. 1, 1218-1225.

72) Shibutani, H., Sakata, H. and Hyvärinen, J. (1984) Saccade and blinking evoked by microstimulation of the posterior parietal association cortex of the monkey. Exp. Brain Res. 55, 1-8.

73) Rosini, F., Serchi, V., Tirelli, L., Di Toro Mammarella, L., Pucci, B., Federico, A. and Rufa, A. (2017) Progression of oculomotor deficit in a patient with posterior cortical atrophy. J. Neurol. Sci. 372, 459-463.

74) Andersen, R.A., Brotchie, P.R. and Mazzoni, P. (1992) Evidence for the lateral intraparietal area as the parietal eye field. Curr. Opin. Neurobiol. 2, 840-846.

75) Duhamel, J.R., Colby, C.L. and Goldberg, M.E. (1992) The updating of the representation of visual space in parietal cortex by intended eye movements. Science 255, 90-92.

76) Gnadt, J.W. and Andersen, R.A. (1988) Memory related motor planning activity in posterior parietal cortex of macaque. Exp. Brain Res. 70, $216-220$.

77) Oyachi, H. and Ohtsuka, K. (1995) Transcranial magnetic stimulation of the posterior parietal cortex degrades accuracy of memory-guided saccades in humans. Invest. Ophthalmol. Vis. Sci. 36, 1441-1449.

78) Robinson, D.A. (1975) Tectal oculomotor connections. Neurosci. Res. Program Bull. 13, 238-244.

79) Pélisson, D. and Prablanc, C. (1988) Kinematics of centrifugal and centripetal saccadic eye movements in man. Vision Res. 28, 87-94.

80) Robinson, F.R., Straube, A. and Fuchs, A.F. (1993) Role of the caudal fastigial nucleus in saccade generation. II. Effects of muscimol inactivation. J. Neurophysiol. 70, 1741-1758.

81) Schmitt, L.M., Cook, E.H., Sweeney, J.A. and Mosconi, M.W. (2014) Saccadic eye movement abnormalities in autism spectrum disorder indicate dysfunctions in cerebellum and brainstem. Mol. Autism 5, 47.

82) Wessel, K., Moschner, C., Wandinger, K.P., Kömpf, D. and Heide, W. (1998) Oculomotor testing in the differential diagnosis of degenerative ataxic disorders. Arch. Neurol. 55, 949-956.

83) Federighi, P., Cevenini, G., Dotti, M.T., Rosini, F., Pretegiani, E., Federico, A. and Rufa, A. (2011) Differences in saccade dynamics between spinocerebellar ataxia 2 and late-onset cerebellar ataxias. Brain 134, 879-891.

84) Filippopulos, F., Eggert, T. and Straube, A. (2013) Deficits of cortical oculomotor mechanisms in cerebellar atrophy patients. Exp. Brain Res. 224, 541-550.

85) Filippopulos, F., Eggert, T. and Straube, A. (2013) Effects of cerebellar infarcts on cortical processing of saccades. J. Neurol. 260, 805-814.

86) Sweeney, J.A., Levy, D. and Harris, M.S. (2002) Commentary: eye movement research with clin- ical populations. Prog. Brain Res. 140, 507-522.

87) Antoniades, C.A. and Kennard, C. (2015) Ocular motor abnormalities in neurodegenerative disorders. Eye (Lond.) 29, 200-207.

88) Kimmig, H., Haussmann, K., Mergner, T. and Lücking, C.H. (2002) What is pathological with gaze shift fragmentation in Parkinson's disease? J. Neurol. 249, 683-692.

89) DeJong, J.D. and Jones, G.M. (1971) Akinesia, hypokinesia, and bradykinesia in the oculomotor system of patients with Parkinson's disease. Exp. Neurol. 32, 58-68.

90) Shibasaki, H., Tsuji, S. and Kuroiwa, Y. (1979) Oculomotor abnormalities in Parkinson's disease. Arch. Neurol. 36, 360-364.

91) Teräväinen, H. and Calne, D.B. (1980) Studies of parkinsonian movement: 1. Programming and execution of eye movements. Acta Neurol. Scand. 62, 137-148.

92) White, O.B., Saint-Cyr, J.A., Tomlinson, R.D. and Sharpe, J.A. (1983) Ocular motor deficits in Parkinson's disease. II. Control of the saccadic and smooth pursuit systems. Brain 106, 571-587.

93) Rascol, O., Clanet, M., Montastruc, J.L., Simonetta, M., Soulier-Esteve, M.J., Doyon, B. and Rascol, A. (1989) Abnormal ocular movements in Parkinson's disease: evidence for involvement of dopaminergic systems. Brain 112, 1193-1214.

94) Vidailhet, M., Rivaud, S., Gouider-Khouja, N., Pillon, B., Bonnet, A.M., Gaymard, B., Agid, Y. and Pierrot-Deseilligny, C. (1994) Eye movements in parkinsonian syndromes. Ann. Neurol. 35, 420426.

95) Albin, R.L., Young, A.B. and Penney, J.B. (1995) The functional anatomy of disorders of the basal ganglia. Trends Neurosci. 18, 63-64.

96) Terao, Y., Fukuda, H., Ugawa, Y. and Hikosaka, O. (2013) New perspectives on the pathophysiology of Parkinson's disease as assessed by saccade performance. A clinical review. Clin. Neurophysiol. 124, 1491-1506.

97) Basso, M.A. and Liu, P. (2007) Context-dependent effects of substantia nigra stimulation on eye movements. J. Neurophysiol. 97, 4129-4142.

98) Neggers, S.F., Raemaekers, M.A., Lampmann, E.E., Postma, A. and Ramsey, N.F. (2005) Cortical and subcortical contributions to saccade latency in the human brain. Eur. J. Neurosci. 21, 2853-2863.

99) van Donkelaar, P., Saavedra, S. and Woollacott, M. (2007) Multiple saccades are more automatic than single saccades. J. Neurophysiol. 97, 3148-3151.

100) Briand, K.A., Strallow, D., Hening, W., Poizner, H. and Sereno, A.B. (1999) Control of voluntary and reflexive saccades in Parkinson's disease. Exp. Brain Res. 129, 38-48.

101) Kingstone, A., Klein, R., Morein-Zamir, S., Hunt, A., Fisk, J. and Maxner, C. (2002) Orienting attention in aging and Parkinson's disease: distinguishing modes of control. J. Clin. Exp. Neuropsychol. 24, 951-967.

102) Roll, A., Wierzbicka, M.M. and Wolf, W. (1996) The "gap paradigm" leads to express-like saccadic 
reaction times in Parkinson's disease. Exp. Brain Res. 111, 131-138.

103) Chan, F., Armstrong, I.T., Pari, G., Riopelle, R.J. and Munoz, D.P. (2005) Deficits in saccadic eye movement control in Parkinson's disease. Neuropsychologia 43, 784-796.

104) Joti, P., Kulashekhar, S., Behari, M. and Murthy, A. (2007) Impaired inhibitory oculomotor control in patients with Parkinson's disease. Exp. Brain Res. 177, 447-457.

105) van Stockum, S., MacAskill, M., Anderson, T. and Dalrymple-Alford, J. (2008) Don't look now or look away: two sources of saccadic disinhibition in Parkinson's disease? Neuropsychologia 46, 31083115 .

106) Kitagawa, M., Fukushima, J. and Tashiro, K. (1994) Relationship between antisaccades and the clinical symptoms in Parkinson's disease. Neurology 44, 2285-2289.

107) van Koningsbruggen, M.G., Pender, T., Machado, L. and Rafal, R.D. (2009) Impaired control of the oculomotor reflexes in Parkinson's disease. Neuropsychologia 47, 2909-2915.

108) Chambers, J.M. and Prescott, T.J. (2010) Response times for visually guided saccades in persons with Parkinson's disease: a meta-analytic review. Neuropsychologia 48, 887-899.

109) Selhorst, J.B., Stark, L., Ochs, A.L. and Hoyt, W.F. (1976) Disorders in cerebellar ocular motor control. I. Saccadic overshoot dysmetria. An oculographic, control system and clinico-anatomical analysis. Brain 99, 497-508.

110) Moschner, C. and Baloh, R.W. (1994) Age-related changes in visual tracking. J. Gerontol. 49, M235M238.

111) Federighi, P., Cevenini, G., Dotti, M.T., Rosini, F., Pretegiani, E., Federico, A. and Rufa, A. (2011) Differences in saccade dynamics between spinocerebellar ataxia 2 and late-onset cerebellar ataxias. Brain 134, 879-891.

112) Kheradmand, A. and Zee, D.S. (2011) Cerebellum and ocular motor control. Front. Neurol. 2, 53.

113) Flaherty, A.W. and Rost, N.S. (2007) The Massachusetts General Hospital Handbook of Neurology. 2nd edition. Lippincott Williams \& Wilkins, Philadelphia.

114) Terao, Y., Fukuda, H., Tokushuge, S., InomataTerada, S., Yugeta, A., Hamada, M. and Ugawa, Y. (2016) Distinguishing spinocerebellar ataxia with pure cerebellar manifestation from multiple system atrophy (MSA-C) through saccade profiles. Clin. Neurophysiol. 128, 31-43.

115) Dean, P. (1995) Modelling the role of the cerebellar fastigial nuclei in producing accurate saccades, the importance of burst timing. Neuroscience 68, 1059-1077.

116) Buzunov, E., Mueller, A., Straube, A. and Robinson, F.R. (2013) When during horizontal saccades in monkey does cerebellar output affect movement? Brain Res. 1503, 33-42.

117) Fuchs, A.F., Robinson, F.R. and Straube, A. (1993) Role of the caudal fastigial nucleus in saccade generation. I. Neuronal discharge pattern. J. Neurophysiol. 170, 1723-1740.

118) Noda, H. (1991) Cerebellar control of saccadic eye movements: its neural mechanisms and pathways. Jpn. J. Physiol. 41, 351-368.

119) Ohtsuka, K. and Noda, H. (1990) Direction-selective saccadic-burst neurons in the fastigial oculomotor region of the macaque. Exp. Brain Res. 81, 659662.

120) Ohtsuka, K. and Noda, H. (1991) Saccadic burst neurons in the oculomotor region of the fastigial nucleus of macaque monkeys. J. Neurophysiol. 65, 1422-1434.

121) Quaia, C., Lefèvre, P. and Optican, L.M. (1999) Model of the control of saccades by superior colliculus and cerebellum. J. Neurophysiol. 82, 999-1018.

122) Ohtsuka, K. and Noda, H. (1992) Burst discharges of fastigial neurons in macaque monkeys are driven by vision- and memory-guided saccades but not by spontaneous saccades. Neurosci. Res. 15, 224-228.

123) Terao, Y., Fukuda, H., Tokushige, S., InomataTerada, S., Yugeta, A., Hamada, M., Ichikawa, Y., Hanajima, R. and Ugawa, Y. (2016) Is multiple system atrophy with cerebellar ataxia (MSA-C) like spinocerebellar ataxia and multiple system atrophy with parkinsonism (MSA-P) like Parkinson's disease? - A saccade study on pathophysiology. Clin. Neurophysiol. 127, 1491-1502.

124) Terao, Y., Fukuda, H., Shirota, Y., Yugeta, A., Yoshioka, M., Suzuki, M., Hanajima, R., Nomura, Y., Segawa, M., Tsuji, S. and Ugawa, Y. (2013) Deterioration of horizontal saccades in progressive supranuclear palsy. Clin. Neurophysiol. 124, 354363.

125) Gorges, M., Pinkhardt, E.H. and Kassubek, J. (2014) Alterations of eye movement control in neurodegenerative movement disorders. J. Ophthalmol. 2014, 658243 .

126) Bhidayasiri, R., Riley, D.E., Somers, J.T., Lerner, A.J., Büttner-Ennever, J.A. and Leigh, R.J. (2001) Pathophysiology of slow vertical saccades in progressive supranuclear palsy. Neurology 57, 2070-2077.

127) Bahill, A.T., Clark, M.R. and Stark, L. (1975) The main sequence, a tool for studying human eye movements. Math. Biosci. 24, 191-204.

128) Otero-Millan, J., Macknik, S.L., Serra, A., Leigh, R.J. and Martinez-Conde, S. (2011) Triggering mechanisms in microsaccade and saccade generation: a novel proposal. Ann. N. Y. Acad. Sci. 1233, 107-116.

129) Otero-Millan, J., Schneider, R., Leigh, R.J., Macknik, S.L. and Martinez-Conde, S. (2013) Saccades during attempted fixation in parkinsonian disorders and recessive ataxia: from microsaccades to square-wave jerks. PLoS One 8, e58535.

130) Yugeta, A., Terao, Y., Fukuda, H., Hikosaka, O., Okiyama, R., Yokochi, F., Taniguchi, M., Takahashi, H., Hamada, I., Hanajima, R. and 
Ugawa, Y. (2010) Effects of STN stimulation on the initiation and inhibition of saccade in Parkinson's disease. Neurology 74, 743-748.

131) Fimm, B., Heber, I.A., Coenen, V.A., Fromm, C., Noth, J. and Kronenbuerger, M. (2009) Deep brain stimulation of the subthalamic nucleus improves intrinsic alertness in Parkinson's disease. Mov. Disord. 24, 1613-1620.

132) Lohnes, C.A. and Earhart, G.M. (2012) Effect of subthalamic deep brain stimulation on turning kinematics and related saccadic eye movements in Parkinson disease. Exp. Neurol. 236, 389-394.

133) Antoniades, C.A., Carpenter, R.H. and Temel, Y. (2012) Deep brain stimulation of the subthalamic nucleus in Parkinson's disease: similar improvements in saccadic and manual responses. Neuroreport 23, 179-183.

134) Antoniades, C.A., Rebelo, P., Kennard, C., Aziz, T.Z., Green, A.L. and FitzGerald, J.J. (2015) Pallidal deep brain stimulation improves higher control of the oculomotor system in Parkinson's disease. J. Neurosci. 35, 13043-13052.

135) Fischer, P., Ossandón, J., Keyser, J., Gulberti, A., Wilming, N., Hamel, W., Köppen, J., Buhmann, C., Westphal, M., Gerloff, C., Moll, C.K., Engel, A.K. and König, P. (2016) STN-DBS reduces saccadic hypometria but not visuospatial bias in Parkinson's disease patients. Front. Behav. Neurosci. 10, 85 .

136) FitzGerald, J.J. and Antoniades, C.A. (2016) Eye movements and deep brain stimulation. Curr. Opin. Neurol. 29, 69-73.

137) Antoniades, C.A. and FitzGerald, J.J. (2016) Using saccadometry with deep brain stimulation to study normal and pathological brain function. J. Vis. Exp. 114, 113.

138) Malhotra, P., Coulthard, E. and Husain, M. (2006) Hemispatial neglect, balance and eye-movement control. Curr. Opin. Neurol. 19, 14-20.

139) Husain, M. (2008) Hemispatial neglect. Handb. Clin. Neurol. 88, 359-372.

140) Duc, A.H., Bays, P. and Husain, M. (2008) Eye movements as a probe of attention. Prog. Brain Res. 171, 403-411.

141) Thurston, S.E., Leigh, R.J., Crawford, T.,
Thompson, A. and Kennard, C. (1988) Two distinct deficits of visual tracking caused by unilateral lesions of cerebral cortex in humans. Ann. Neurol. 23, 266-273.

142) Rayner, K. (2009) Eye movements and landing positions in reading: a retrospective. Perception 38, 895-899.

143) Cohen, L., Henry, C., Dehaene, S., Martinaud, O., Lehéricy, S., Lemer, C. and Ferrieux, S. (2004) The pathophysiology of letter-by-letter reading. Neuropsychologia 42, 1768-1780.

144) Foulsham, T., Barton, J.J., Kingstone, A., Dewhurst, R. and Underwood, G. (2009) Fixation and saliency during search of natural scenes: the case of visual agnosia. Neuropsychologia 47, 1994-2003.

145) Mosimann, U.P., Felblinger, J., Ballinari, P., Hess, C.W. and Müri, R.M. (2004) Visual exploration behaviour during clock reading in Alzheimer's disease. Brain 127, 431-438.

146) Spezio, M.L., Huang, P.Y., Castelli, F. and Adolphs, R. (2007) Amygdala damage impairs eye contact during conversations with real people. J. Neurosci. 27, 3994-3997.

147) Adolphs, R., Gosselin, F., Buchanan, T.W., Tranel, D., Schyns, P. and Damasio, A.R. (2005) A mechanism for impaired fear recognition after amygdala damage. Nature 433, 68-72.

148) Moriuchi, J.M., Klin, A. and Jones, W. (2017) Mechanisms of diminished attention to eyes in autism. Am. J. Psychiatry 174, 26-35.

149) Matsumoto, H., Terao, Y., Furubayashi, T., Yugeta, A., Fukuda, H., Emoto, M., Hanajima, R. and Ugawa, Y. (2011) Small saccades restrict visual scanning area in Parkinson's disease. Mov. Disord. 26, 1619-1626.

150) Tseng, P.H., Cameron, I.G., Pari, G., Reynolds, J.N., Munoz, D.P. and Itti, L. (2013) Highthroughput classification of clinical populations from natural viewing eye movements. J. Neurol. 260, 275-284.

(Received July 5, 2017; accepted Aug. 17, 2017) 


\section{Profile}

Yasuo Terao was born in Shizuoka Prefecture in 1964 and graduated from the School of Medicine, the University of Tokyo in 1989. After being trained in internal medicine, he has majored in neurology since 1991. After working as a neurologist at the Department of Neurology of medical centers such as the Japanese Red Cross Medical Center, Tokyo Metropolitan Neurological Hospital, and the University of Tokyo Hospital, he received his Ph.D. at the Graduate School of Medicine, the University of Tokyo, in 1998. He worked as a postdoctoral fellow at the Department of Physiology, Umeå University, Sweden between 1999 and 2001 with Professor Roland S. Johansson. He became Lecturer of Medicine at the Department of Neurology, the University of Tokyo in 2012, and Professor of Physiology (Department of Cell Physiology) at Kyorin

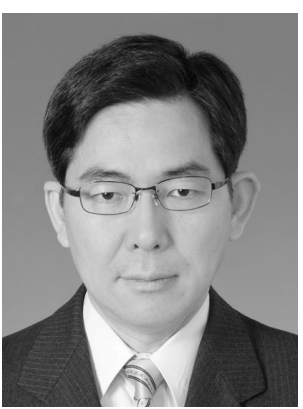
University in 2016. As a clinical neurophysiologist, he has been working on clinical studies of neurological patients using saccade recording and eye tracking methodologies and human studies using various electrophysiological techniques, such as transcranial magnetic stimulation and magnetoencephalography. His main interest is in elucidating the neural mechanisms for voluntary motor function in normal humans as well as the pathophysiological bases of neurological disorders.

\section{Profile}

Okihide Hikosaka received his M.D. and Ph.D. from the University of Tokyo in 1978, where he worked in the laboratory of Professor Hiroshi Shimazu on the brainstem vestibulo-oculomotor system. He carried out postdoctoral research with Dr. Robert Wurtz at the Laboratory of Sensorimotor Research, NEI (1979-1982) on the role of the substantia nigra pars reticulata in the control of saccadic eye movements. He became Assistant Professor at Toho University School of Medicine (Tokyo) in 1979, Full Professor at the National Institute of Physiological Sciences (Okazaki) in 1988, and Full Professor at the Juntendo University School of Medicine (Tokyo) in 1993. In 2002, he returned to the Laboratory of Sensorimotor Research, NEI as a Senior Investigator and Chief of the Section of Neuronal Networks. He received the Tsukahara Memorial Award

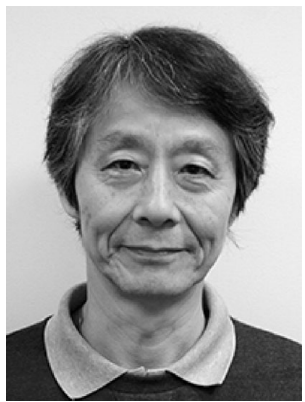
in 1989, Tokizane Toshihiko Memorial Award in 1999, Golden Brain Award in 2015, and NIH Director Award in 2016. He was elected to the American Academy of Arts \& Sciences in 2011 and was a NIH Distinguished Investigator in 2016. His main research interests lie in the neural mechanisms of voluntary behavior. His laboratory studies the mechanisms of motivation, learning, skill, decision-making, attention, and oculomotor control. 